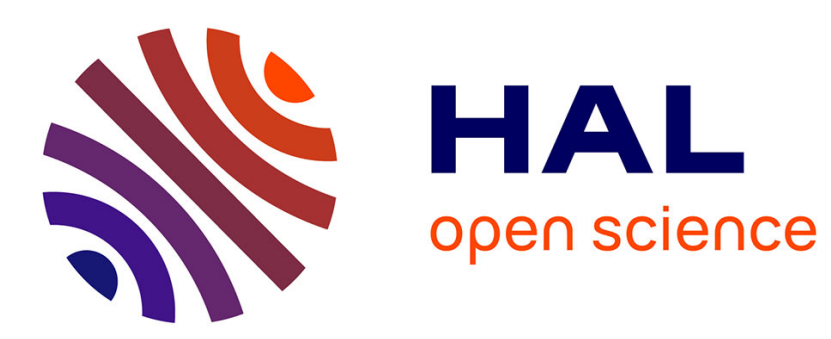

\title{
Hamiltonian regularisation of the unidimensional barotropic Euler equations
}

\author{
Billel Guelmame, Didier Clamond, Stéphane Junca
}

\section{To cite this version:}

Billel Guelmame, Didier Clamond, Stéphane Junca. Hamiltonian regularisation of the unidimensional barotropic Euler equations. Nonlinear Analysis: Real World Applications, 2022, 64, 22p. 10.1016/j.nonrwa.2021.103455 . hal-02907304

\section{HAL Id: hal-02907304 \\ https://hal.science/hal-02907304}

Submitted on 27 Jul 2020

HAL is a multi-disciplinary open access archive for the deposit and dissemination of scientific research documents, whether they are published or not. The documents may come from teaching and research institutions in France or abroad, or from public or private research centers.
L'archive ouverte pluridisciplinaire $\mathbf{H A L}$, est destinée au dépôt et à la diffusion de documents scientifiques de niveau recherche, publiés ou non, émanant des établissements d'enseignement et de recherche français ou étrangers, des laboratoires publics ou privés. 


\title{
HAMILTONIAN REGULARISATION OF THE UNIDIMENSIONAL BAROTROPIC EULER EQUATIONS
}

\author{
BILLEL GUELMAME, DIDIER CLAMOND AND STÉPHANE JUNCA
}

\begin{abstract}
Recently, a Hamiltonian regularised shallow water (Saint-Venant) system has been introduced by Clamond and Dutykh [13]. This system is Galilean invariant, linearly non-dispersive and conserves formally an $H^{1}$-like energy. In this paper, we generalise this regularisation for the barotropic Euler system preserving the same properties. We prove the local (in time) well-posedness of the regularised barotropic Euler system and a periodic generalised two-component Hunter-Saxton system. We also show for both systems that if singularities appear in finite time, they are necessary in the first derivatives.
\end{abstract}

AMS Classification: 35Q35; 35L65; 37K05; 35B65; 76B15.

Key words: Barotropic fluids; Euler system; nonlinear hyperbolic systems; generalised two-component Hunter-Saxton system; regularisation; Hamiltonian; energy conservation; dispersionless.

\section{CONTENTS}

1. Introduction 1

2. Equations for barotropic perfect fluids 3

3. Regularised barotropic flows 6

4. Local well-posedness of the regularised barotropic Euler system 11

5. A generalised two-component Hunter-Saxton system 19

6. Remarks on a special regularision 20

7. Conclusion and perspectives 22

Appendix A. Isentropic flows 22

References 23

\section{INTRODUCTION}

The barotropic Euler system is a quasilinear system of partial differential equations that can be used to describe many phenomena in fluid mechanics. Denoting the time and the spacial coordinate by the independent variables $t$ and $x$, respectively, and denoting the density, the velocity and the pressure by the dependent variables $\rho(t, x)>0, u(t, x)$ and $P(\rho)$, respectively, the conservation of mass and momentum yield

$$
\rho_{t}+[\rho u]_{x}=0, \quad u_{t}+u u_{x}+P_{x} / \rho=0,
$$


where subscripts denote partial derivatives. If the pressure $P$ is an increasing function of $\rho$, the system (1) is hyperbolic and, even for smooth initial data, the solutions may develop shocks in finite time. In order to avoid those shocks, several regularisations have been proposed, for example by adding a "small" artificial viscosity and/or dispersive terms $[7,16,24,31,33,34,35,47]$. The artificial viscosity leads to a loss of the energy everywhere and the dispersive terms lead to high oscillations which cause problems in numerical computations. Other regularisations of Leray-type (for Burgers equation, isentropic Euler system and others) have been proposed and studied in [3, 4, 5, 6, 11, 41, 42]. Those regularisations do not conserve the energy and the limit solution fails (in general) to satisfy the Lax entropy condition [4].

Modifying the dispersion of the Serre-Green-Naghdi system, Clamond and Dutykh [13] proposed the dispersionless regularised Saint-Venant (shallow water) system

$$
\begin{array}{r}
h_{t}+[h u]_{x}=0, \\
{[h u]_{t}+\left[h u^{2}+\frac{1}{2} g h^{2}+\epsilon \mathscr{R}\right]_{x}=0,} \\
\mathscr{R} \stackrel{\text { def }}{=} 2 h^{3} u_{x}^{2}-h^{3}\left[u_{t}+u u_{x}+g h_{x}\right]_{x}-\frac{1}{2} g h^{2} h_{x}^{2},
\end{array}
$$

where $h$ is the total water depth of the fluid, $g$ is the gravitational acceleration and $\varepsilon>0$ is a dimensionless parameter. This Hamiltonian regularisation conserves an $H^{1}$-like energy for smooth solutions and it has the same shock speed as the classical Saint-Venant system. Weak singular shocks of (2) have been studied by Pu et al. [45]. Also, local (in time) wellposedness and existence of blowing-up solutions using Ricatti-type equations have been proved in [36]. The global well-posedness and a mathematical study of the case $\varepsilon \rightarrow 0$ remains open problems. Recently, inspired by [13] and with the same properties as (2), a similar regularisation has been proposed for the inviscid Burgers equation in [23] and for general scalar conservation laws in [22], where solutions exist globally (in time) in $H^{1}$, those solutions converging to solutions of the classical equation when $\varepsilon \rightarrow 0$ at least for a short time [22, 23]. The regularised Saint-Venant system (2) has been also generalised for shallow water equations with uneven bottom [14].

The classical Saint-Venant system (letting $\varepsilon \rightarrow 0$ in (2)) is, formally, a special case of the barotropic Euler system (1) such that $\rho \equiv h$ and $P(\rho) \equiv g h^{2} / 2$ (i.e., isentropic Euler equation with $\gamma=2$, see Appendix A). The aim of this paper is to generalise the system (2) to regularise the barotropic Euler system (1) as in [13], that is preserving the same properties. In Section 3 below, modifying the Lagrangian of (1), we obtain the regularised barotropic Euler (rbE) system

$$
\begin{array}{r}
\rho_{t}+[\rho u]_{x}=0, \\
{[\rho u]_{t}+\left[\rho u^{2}+\rho \mathscr{V}^{\prime}-\mathscr{V}+\epsilon \mathscr{R}\right]_{x}=0,} \\
\mathscr{R} \stackrel{\text { def }}{=}\left(\rho^{2} \mathscr{A}^{\prime}\right)^{\prime} u_{x}^{2}-2 \rho \mathscr{A}^{\prime}\left[u_{t}+u u_{x}+\varpi_{x}\right]_{x}+\left(\rho^{\prime \prime} / \mathscr{A}^{\prime}\right)^{\prime} \mathscr{A}_{x}^{2},
\end{array}
$$

where primes denote derivatives with respect to $\rho, \mathscr{V}^{\prime \prime}(\rho)=P^{\prime}(\rho) / \rho$ and $\mathscr{A}$ is a smooth increasing function of $\rho$. We show in this paper that the system (3) is non-dispersive, non-diffusive, it has a Hamiltonian structure, it has the same shock speed as (1) and, for 
all $\mathscr{A}$ smooth increasing function of $\rho$, smooth solutions of (3) conserve an $H^{1}$-like energy. A study of steady solutions of rbE has been also done, which covers the traveling waves due to the Galilean invariance of rbE.

Introducing the linear Sturm-Liouville operator

$$
\mathcal{L}_{\rho} \stackrel{\text { def }}{=} \rho-2 \epsilon \partial_{x} \rho \mathscr{A}^{\prime} \partial_{x},
$$

and applying $\mathcal{L}_{\rho}^{-1}$ on $(3 \mathrm{~b})$ (the invertibility of $\mathcal{L}_{\rho}$ is insured by Lemma 3 below), the system (3) becomes

$$
\begin{aligned}
\rho_{t}+[\rho u]_{x} & =0 \\
u_{t}+u u_{x}+P_{x} / \rho & =-\epsilon \mathcal{L}_{\rho}^{-1} \partial_{x}\left\{\left(\rho^{2} \mathscr{A}^{\prime}\right)^{\prime} u_{x}^{2}+\left(\rho \mathscr{V}^{\prime \prime} / \mathscr{A}^{\prime}\right)^{\prime} \mathscr{A}^{\prime 2} \rho_{x}^{2}\right\} .
\end{aligned}
$$

The reason of applying $\mathcal{L}_{\rho}^{-1}$ is to remove the derivative with respect to $t$ and the high-order derivatives with respect to $x$ appearing in (3c). The form (5) is then more convenient to obtain the local well-posedness of rbE. Following [1, 2, 27, 36, 39], we prove that if the initial data is an $H^{s}$ perturbation of a constant state (with $s \geqslant 2$, and $\rho \geqslant \rho^{*}>0$ ), then (5) is locally well-posed. The same proof is used to prove the local existence of periodic solutions of the generalised two-component Hunter-Saxton system

$$
\begin{aligned}
\rho_{t}+[\rho u]_{x} & =0, \\
u_{t}+u u_{x}+P_{x} / \rho & =\partial_{x}^{-1}\left\{\left(1+\frac{\rho \mathscr{A}^{\prime \prime}}{2 \mathscr{A}^{\prime}}\right) u_{x}^{2}+\left(\frac{\left(\rho \mathscr{V}^{\prime \prime}\right)^{\prime}}{2 \rho}-\frac{\mathscr{V}^{\prime \prime} \mathscr{A}^{\prime \prime}}{2 \mathscr{A}^{\prime}}\right) \rho_{x}^{2}\right\},
\end{aligned}
$$

that can be obtained by formally taking $\varepsilon \rightarrow \infty$ in (5).

This paper is divided on two parts. A first part (sections 2,3 ) presents the physical motivations of the regularised barotropic Euler system and its properties. A second part (sections 4, 5) consist on mathematical proofs for existence results. Shortly speaking, the first part is more physical and the second one is more mathematical. More specifically, the content of the paper is organised as follows. In Section 2, we recall some classical properties of the barotropic Euler system (1). Section 3 is devoted to derive the regularised system (3), study its properties and steady motions. In Section 4, we prove the local well-posedness and a blow-up criteria of (5). In Section 5, the generalised Hunter-Saxton system (6) is introduced, and a well-posedness theorem is given. A special choice of the regularising function $\mathscr{A}$, with some interesting properties, is briefly discussed in Section 6 .

\section{EQUATIONS FOR BAROTROPIC PERFECT FLUIDS}

Let us recall the conservation of mass and momentum for perfect fluids in Eulerian description of motion

$$
\begin{aligned}
\rho_{t}+[\rho u]_{x} & =0, \\
u_{t}+u u_{x}+P_{x} / \rho & =0 .
\end{aligned}
$$

Note that (conservative) body forces, if present, are incorporated into the definition of the pressure $P$. In the special case of barotropic motions [46] - i.e., when $\rho=\rho(P)$ or 
$P=P(\rho)$ - it is convenient to introduce the so-called specific enthalpy $[18, \S 3.3] \varpi$ such that

$$
\varpi=\int \frac{\mathrm{d} P}{\rho(P)}=\int \frac{\mathrm{d} P(\rho)}{\mathrm{d} \rho} \frac{\mathrm{d} \rho}{\rho} \quad \Rightarrow \quad \rho \mathrm{d} \varpi=\mathrm{d} P, \quad \partial_{x} \varpi=\frac{\partial_{x} P}{\rho} .
$$

$\varpi$ being an antiderivative of $1 / \rho(P)$, it is defined modulo an additional arbitrary integration constant, so the value of $\varpi$ can be freely chosen on a given isobaric surface $P=$ constant (thus providing gauge condition for the specific enthalpy). The relation (8) can also be written in the reciprocal form $P(\varpi)=\int \rho(\varpi) \mathrm{d} \varpi$, thence $P$ is a known function of $\varpi$ and, obviously, $P=\rho \varpi$ if the density is constant. The speed of sound $c_{s}$ is defined by

$$
c_{s} \stackrel{\text { def }}{=}[\mathrm{d} \rho / \mathrm{d} P]^{-\frac{1}{2}}=\left[\rho^{-1} \mathrm{~d} \rho / \mathrm{d} \varpi\right]^{-\frac{1}{2}} .
$$

From this definition we have $\rho \mathrm{d} \varpi=c_{s}^{2} \mathrm{~d} \rho$, thence with the mass conservation (7a)

$$
\mathrm{D}_{t} \varpi=\rho^{-1} c_{s}^{2} \mathrm{D}_{t} \rho=-c_{s}^{2} u_{x}
$$

where $\mathrm{D}_{t} \stackrel{\text { def }}{=} \partial_{t}+u \partial_{x}$ is the temporal derivative following the motion. The relation (10) is of special interest when $c_{s}$ is constant. Many equations of state for compressible fluids can be found in the literature [49]. Isentropic motions are of special interest so their equation of state is given in Appendix A.

2.1. Cauchy-Lagrange equation. For barotropic fluids, the momentum equation (7b) becomes

$$
u_{t}+u u_{x}+\varpi_{x}=0,
$$

and introducing a velocity potential $\phi$ such that $u=\phi_{x}$, the equation (11) is integrated into a Cauchy-Lagrange equation

$$
\phi_{t}+\frac{1}{2} \phi_{x}^{2}+\varpi=K(t) \equiv 0,
$$

where $K(t)$ is an integration constant that can be set to zero without loose of generality (gauge condition for the velocity potential).

2.2. Conservation laws. For regular solutions, secondary conservation laws can be derived from (7), e.g.,

$$
\begin{aligned}
{[\rho u]_{t}+\left[\rho u^{2}+\rho^{\mathscr{V}^{\prime}}-\mathscr{V}\right]_{x} } & =0, \\
{[u]_{t}+\left[\frac{1}{2} u^{2}+\mathscr{V}^{\prime}\right]_{x} } & =0, \\
{\left[\frac{1}{2} \rho u^{2}+\mathscr{V}\right]_{t}+\left[\left(\frac{1}{2} \rho u^{2}+\rho \mathscr{V}^{\prime}\right) u\right]_{x} } & =0, \\
{\left[\frac{1}{6} \rho u^{3}+\mathscr{V} u\right]_{t}+\left[\frac{1}{2}\left(\frac{1}{3} \rho u^{2}+\rho \mathscr{V}^{\prime}+\mathscr{V}\right) u^{2}+\mathscr{W}_{1}\right]_{x} } & =0, \\
{\left[\frac{1}{24} \rho u^{4}+\frac{1}{2} \mathscr{V} u^{2}+\mathscr{W}_{3}\right]_{t}+\left[\frac{1}{3}\left(\frac{1}{8} \rho u^{2}+\frac{1}{2} \rho \mathscr{V}^{\prime}+\mathscr{V}\right) u^{3}+\mathscr{W}_{2} u\right]_{x} } & =0,
\end{aligned}
$$

where

$$
\mathscr{V} \stackrel{\text { def }}{=} \int \varpi \mathrm{d} \rho, \quad \mathscr{W}_{1} \stackrel{\text { def }}{=} \int \mathscr{V} \mathscr{V}^{\prime \prime} \mathrm{d} \rho, \quad \mathscr{W}_{2} \stackrel{\text { def }}{=} \rho \int \frac{\mathscr{V}^{\prime \prime}}{\rho} \mathrm{d} \rho, \quad \mathscr{W}_{3} \stackrel{\text { def }}{=} \int \frac{\mathscr{W}_{2}}{\rho} \mathrm{d} \rho .
$$


Actually, since the barotropic Euler system is a $2 \times 2$ strictly hyperbolic system, an infinite number of conservation laws can be derived [17, 32].

2.3. Jump conditions. The Euler equations admit weak solutions. For discontinuous $\rho$ and $u$, the Rankine-Hugoniot conditions for the mass and momentum conservation are

$$
(u-\dot{s}) \llbracket \rho \rrbracket+\rho \llbracket u \rrbracket=0, \quad(u-\dot{s}) \llbracket u \rrbracket+\varpi^{\prime} \llbracket \rho \rrbracket=0,
$$

where $\varpi^{\prime}=\mathrm{d} \varpi / \mathrm{d} \rho, \dot{s} \stackrel{\text { def }}{=} \mathrm{d} s / \mathrm{d} t$ is the speed of the shock located at $x=s(t)$ and $\llbracket f \rrbracket \stackrel{\text { def }}{=}$ $f\left(x=s^{+}\right)-f\left(x=s^{-}\right)$denotes the jump across the shock for any function $f$. The RankineHugoniot conditions (18) yield at once the shock speed

$$
\dot{s}(t)=u \pm \sqrt{\rho \varpi^{\prime}} \quad \text { at } \quad x=s(t) .
$$

A goal of the present work is to derive a regularisation of the Euler equation that preserves exactly this shock speed.

2.4. Variational formulations. An interesting feature of the equations above is that they can be derived from a variational principle. Indeed, the (so-called action) functional $S=\int_{t_{1}}^{t_{2}} \int_{x_{1}}^{x_{2}} \mathscr{L} \mathrm{d} x \mathrm{~d} t$ with the Lagrangian density

$$
\mathscr{L} \stackrel{\text { def }}{=} \rho \phi_{t}+\frac{1}{2} \rho \phi_{x}^{2}+\mathscr{V}(\rho),
$$

where $\mathscr{V}$ is the density of potential energy defined by

$$
\mathscr{V}(\rho) \stackrel{\text { def }}{=} \int \varpi(\rho) \mathrm{d} \rho,
$$

provided that an equation of state $\varpi(\rho)$ (such as (148) given in Appendix A), is substituted into the right-hand side of $(21)$. Since $\varpi(\bar{\rho})=0$ with (148) ( $\bar{\rho}$ a constant state of reference), $\mathscr{V}$ is such that $\mathscr{V}^{\prime}(\bar{\rho})=0$. Note that $\mathscr{V}$ can also be kept explicitly into the Lagrangian if the equation of stated is added via a Lagrange multiplier $\lambda$, i.e., considering the Lagrangian density

$$
\mathscr{L}^{\prime} \stackrel{\text { def }}{=} \mathscr{L}+\left\{\mathscr{V}(\rho)-\int \varpi(\rho) \mathrm{d} \rho\right\} \lambda .
$$

This is of no interest here, however, so we do not consider this generalisation, for simplicity. The Euler-Lagrange equations for the Lagrangian density (20) yield

$$
\begin{aligned}
& \delta \phi: \quad 0=\rho_{t}+\left[\rho \phi_{x}\right]_{x}, \\
& \delta \rho: \quad 0=\phi_{t}+\frac{1}{2} \phi_{x}^{2}+\mathscr{V}^{\prime}(\rho),
\end{aligned}
$$

so the equations of motion (7a) and (12) are recovered.

An alternative variational formulation is obtained from the Hamilton principle yielding the Lagrangian density

$$
\mathscr{L}_{0} \stackrel{\text { def }}{=} \frac{1}{2} \rho u^{2}-\mathscr{V}(\rho)+\left\{\rho_{t}+[\rho u]_{x}\right\} \phi,
$$


that is the kinetic minus potential energies plus a constraint enforcing the mass conservation. The Euler-Lagrange equations for (25) yield

$$
\begin{aligned}
\delta \phi: & 0=\rho_{t}+[\rho u]_{x}, \\
\delta u: & 0=u-\phi_{x}, \\
\delta \rho: & 0=\frac{1}{2} u^{2}-\mathscr{V}^{\prime}(\rho)-\phi_{t}-u \phi_{x},
\end{aligned}
$$

and, substituting $\varpi$ for $\mathscr{V}^{\prime}$, the barotropic equations (7a) and (11) are recovered.

The two variational principles above differ by boundary terms only, i.e.,

$$
\mathscr{L}+\mathscr{L}_{0}=[\rho \phi]_{t}+[\rho u \phi]_{x}+\frac{1}{2} \rho\left(u-\phi_{x}\right)^{2},
$$

so the right-hand side yields only boundary terms since $u=\phi_{x}$. As advocated by Clamond and Dutykh [12], the variational Hamilton principle is more useful for practical applications; this point is illustrated in the section 3 below.

\section{Regularised BAROtRopic FlOWS}

Here, we seek for a regularisation of the barotropic Euler equations. We give some heuristic arguments for the derivation of such models.

3.1. Modified Lagrangian. Following the regularisation of Clamond and Dutykh [13] for the Saint-Venant shallow water equations, we seek for a regularisation of the barotropic Euler equation modifying the Lagrangian density as

$$
\overline{\mathscr{L}}_{\epsilon} \stackrel{\text { def }}{=} \mathscr{L}_{0}+\epsilon \mathscr{A}(\rho)\left[u_{t}+u u_{x}\right]_{x}+\epsilon \mathscr{B}(\rho)\left[\mathscr{V}^{\prime \prime}(\rho) \rho_{x}\right]_{x},
$$

where $\epsilon \geqslant 0$ is a real parameter at our disposal and $\mathscr{A}$ and $\mathscr{B}$ are functions of $\rho$ to be chosen later with suitable properties.

Note that we could also seek for modifications separating $u_{t}$ and $u u_{x}$ in the additional terms - i.e., replacing $\epsilon \mathscr{A}(\rho)\left[u_{t}+u u_{x}\right]_{x}$ by $\epsilon \mathscr{A}(\rho) u_{x t}+\epsilon \mathscr{C}(\rho)\left[u u_{x}\right]_{x}$ - but that would break the Galilean invariance. So, $\mathscr{C}=\mathscr{A}$ is the only physically admissible possibilities.

Exploiting the relations

$$
\begin{aligned}
\mathscr{A}(\rho)\left[u_{t}+u u_{x}\right]_{x} & =\left[\mathscr{A}(\rho) u_{x}\right]_{t}+\left[\mathscr{A}(\rho) u u_{x}\right]_{x}+\mathscr{A}^{\prime}(\rho) \rho u_{x}^{2}, \\
\mathscr{B}(\rho)\left[\mathscr{V}^{\prime \prime}(\rho) \rho_{x}\right]_{x} & =\left[\mathscr{B}(\rho) \mathscr{V}^{\prime \prime}(\rho) \rho_{x}\right]_{x}-\mathscr{B}^{\prime}(\rho) \mathscr{V}^{\prime \prime}(\rho) \rho_{x}^{2},
\end{aligned}
$$

we derive the equivalent simplified Lagrangian density

$$
\mathscr{L}_{\epsilon} \stackrel{\text { def }}{=} \frac{1}{2} \rho u^{2}+\epsilon \mathscr{A}^{\prime} \rho u_{x}^{2}-\mathscr{V}-\epsilon \mathscr{B}^{\prime} \mathscr{V}^{\prime \prime} \rho_{x}^{2}+\left\{\rho_{t}+[\rho u]_{x}\right\} \phi .
$$

The functionals given by $\mathscr{L}_{\epsilon}$ and $\overline{\mathscr{L}}_{\epsilon}$ differing only by boundary terms (i.e., $\mathscr{L}_{\epsilon}-\overline{\mathscr{L}}_{\epsilon}=$ $\left.[\cdots]_{t}+[\cdots]_{x}\right)$, they yield the same equations of motion.

From (33), the regularised kinetic and potential energy densities, respectively $\mathscr{K}_{\epsilon}$ and $\mathscr{V}_{\epsilon}$, are

$$
\mathscr{K}_{\epsilon} \stackrel{\text { def }}{=} \frac{1}{2} \rho u^{2}+\epsilon \mathscr{A}^{\prime} \rho u_{x}^{2}, \quad \mathscr{V}_{\epsilon} \stackrel{\text { def }}{=} \mathscr{V}+\epsilon \mathscr{B}^{\prime} \mathscr{V}^{\prime \prime} \rho_{x}^{2} .
$$

The total energy is then

$$
\mathscr{H}_{\epsilon} \stackrel{\text { def }}{=} \frac{1}{2} \rho u^{2}+\epsilon \mathscr{A}^{\prime} \rho u_{x}^{2}+\mathscr{V}+\epsilon \mathscr{B}^{\prime} \mathscr{V}^{\prime \prime} \rho_{x}^{2} .
$$


Note that these energies are positive for all $\epsilon \geqslant 0$ if $\mathscr{A}, \mathscr{B}$ and $\mathscr{V}^{\prime}$ are increasing functions of $\rho$.

3.2. Linearised equations. Here, we consider small perturbations around the rest state $\rho=\bar{\rho}, u=0$ and $\phi=0, \bar{\rho}$ being a positive constant. Introducing $\rho=\bar{\rho}+\tilde{\rho}, u \approx \tilde{u}$, $\phi \approx \tilde{\phi}$, and $f_{0} \stackrel{\text { def }}{=} f(\bar{\rho})$ for any function $f$, the tilde quantities being assumed small, an approximation of $\mathscr{L}_{\epsilon}$ up to the second-order is

$$
\widetilde{\mathscr{L}_{\epsilon}}=\frac{1}{2} \bar{\rho} \tilde{u}^{2}+\epsilon \mathscr{A}_{0}^{\prime} \bar{\rho} \tilde{u}_{x}^{2}-\mathscr{V}_{0}-\frac{1}{2} \mathscr{V}_{0}^{\prime \prime} \tilde{\rho}^{2}-\epsilon \mathscr{B}_{0}^{\prime} \mathscr{V}_{0}^{\prime \prime} \tilde{\rho}_{x}^{2}+\left(\tilde{\rho}_{t}+\bar{\rho} \tilde{u}_{x}\right) \tilde{\phi} .
$$

The Euler-Lagrange (linear) equations for this approximate Lagrangian are

$$
\begin{array}{ll}
\delta \tilde{\phi}: \quad 0=\tilde{\rho}_{t}+\bar{\rho} \tilde{u}_{x}, \\
\delta \tilde{u}: \quad 0=\tilde{u}-2 \epsilon \mathscr{A}_{0}^{\prime} \tilde{u}_{x x}-\tilde{\phi}_{x}, \\
\delta \tilde{\rho}: \quad 0=\mathscr{V}_{0}^{\prime \prime} \tilde{\rho}-2 \epsilon \mathscr{B}_{0}^{\prime} \mathscr{V}_{0}^{\prime \prime} \tilde{\rho}_{x x}+\tilde{\phi}_{t} .
\end{array}
$$

Looking for traveling waves of the form $\tilde{\rho}=R \cos (k x-\omega t), \tilde{u}=U \cos (k x-\omega t)$ and $\tilde{\phi}=\Phi \sin (k x-\omega t)$, the equations (37)-(39) yield $\Phi=\left(1+2 \epsilon k^{2} \mathscr{A}_{0}^{\prime}\right) U / k, U=\omega R / k \bar{\rho}$ and the dispersion relation

$$
\frac{\omega^{2}}{k^{2}}=\bar{\rho} \mathscr{V}_{0}^{\prime \prime} \frac{1+2 \epsilon k^{2} \mathscr{B}_{0}^{\prime}}{1+2 \epsilon k^{2} \mathscr{A}_{0}^{\prime}}
$$

If $\epsilon=0$ the wave is dispersionless, i.e., the phase velocity $c \stackrel{\text { def }}{=} \omega / k$ is independent of the wave number $k$. If $\epsilon>0$, the wave is dispersionless if $\mathscr{B}_{0}^{\prime}=\mathscr{A}_{0}^{\prime}$. This condition should be satisfied for all $\bar{\rho}$ and for all possible (barotropic) equation of state. Thus, we should take

$$
\mathscr{B}(\rho)=\mathscr{A}(\rho) \text {. }
$$

Hereafter, we consider only the special case (41) because we are only interested by nondispersive regularisations of the barotropic Euler equations.

3.3. Equations of motion. With (41), the Euler-Lagrange equations for the Lagrangian density (33) yield

$$
\begin{aligned}
\delta \phi: \quad 0= & \rho_{t}+[\rho u]_{x}, \\
\delta u: \quad 0= & \rho u-2 \epsilon\left[\mathscr{A}^{\prime} \rho u_{x}\right]_{x}-\rho \phi_{x}, \\
\delta \rho: \quad 0= & \frac{1}{2} u^{2}+\epsilon\left(\mathscr{A}^{\prime}+\mathscr{A}^{\prime \prime} \rho\right) u_{x}^{2}-\mathscr{V}^{\prime}+\epsilon\left(\mathscr{A}^{\prime \prime} \mathscr{V}^{\prime \prime}+\mathscr{A}^{\prime} \mathscr{V}^{\prime \prime \prime}\right) \rho_{x}^{2} \\
& +2 \epsilon \mathscr{A}^{\prime} \mathscr{V}^{\prime \prime} \rho_{x x}-\phi_{t}-u \phi_{x},
\end{aligned}
$$

thence

$$
\begin{aligned}
\phi_{x}= & u-2 \epsilon \rho^{-1}\left[\mathscr{A}^{\prime} \rho u_{x}\right]_{x}, \\
\phi_{t}= & -\frac{1}{2} u^{2}+\epsilon\left(\mathscr{A}^{\prime}+\mathscr{A}^{\prime \prime} \rho\right) u_{x}^{2}-\mathscr{V}^{\prime}+\epsilon\left(\mathscr{A}^{\prime \prime} \mathscr{V}^{\prime \prime}+\mathscr{A}^{\prime} \mathscr{V}^{\prime \prime \prime}\right) \rho_{x}^{2} \\
& +2 \epsilon \mathscr{A}^{\prime} \mathscr{V}^{\prime \prime} \rho_{x x}+2 \epsilon u \rho^{-1}\left[\mathscr{A}^{\prime} \rho u_{x}\right]_{x} .
\end{aligned}
$$


Eliminating $\phi$ between these last two relations one obtains

$$
\begin{aligned}
& 0=\partial_{t}\left\{u-2 \epsilon \rho^{-1}\left[\mathscr{A}^{\prime} \rho u_{x}\right]_{x}\right\}+\partial_{x}\left\{\frac{1}{2} u^{2}+\mathscr{V}^{\prime}-2 \epsilon \mathscr{A}^{\prime} \mathscr{V}^{\prime \prime} \rho_{x x}\right. \\
& \left.-\epsilon\left(\mathscr{A}^{\prime}+\mathscr{A}^{\prime \prime} \rho\right) u_{x}^{2}-\epsilon\left(\mathscr{A}^{\prime \prime} \mathscr{V}^{\prime \prime}+\mathscr{A}^{\prime} \mathscr{V}^{\prime \prime \prime}\right) \rho_{x}^{2}-2 \epsilon u \rho^{-1}\left[\mathscr{A}^{\prime} \rho u_{x}\right]_{x}\right\} .
\end{aligned}
$$

The equations (42) and (47) form the regularised Euler equations for barotropic motions studied in the present paper.

3.4. Secondary equations. From the regularised barotropic Euler (rbE) equations (42) and (47), several secondary equations can be derived; in particular:

$$
\begin{aligned}
& u_{t}+u u_{x}+\varpi_{x}+\epsilon \rho^{-1} \mathscr{R}_{x}=0, \\
& {[\rho u]_{t}+\left[\rho u^{2}+\rho \mathscr{V}^{\prime}-\mathscr{V}+\epsilon \mathscr{R}\right]_{x} }=0, \\
& m_{t}+\left[u m+\rho^{\prime}-\mathscr{V}-\epsilon\left(\rho^{2} \mathscr{A}^{\prime}\right)^{\prime} u_{x}^{2}-2 \epsilon \rho \mathscr{A}^{\prime} \varpi_{x x}+\epsilon\left(\rho^{\prime \prime} / \mathscr{A}^{\prime}\right)^{\prime} \mathscr{A}_{x}^{2}\right]_{x}=0, \\
& {\left[\frac{1}{2} \rho u^{2}+\epsilon \rho \mathscr{A}^{\prime} u_{x}^{2}+\mathscr{V}+\epsilon \mathscr{A}^{\prime} \mathscr{V}^{\prime \prime} \rho_{x}^{2}\right]_{t}+} \\
& {\left[\left(\frac{1}{2} \rho u^{2}+\rho \mathscr{V}^{\prime}+\epsilon \rho \mathscr{A}^{\prime} u_{x}^{2}+\epsilon \mathscr{A}^{\prime} \mathscr{V}^{\prime \prime} \rho_{x}^{2}+\epsilon \mathscr{R}\right) u+2 \epsilon \rho \mathscr{A}^{\prime} \mathscr{V}^{\prime \prime} \rho_{x} u_{x}\right]_{x}=0, }
\end{aligned}
$$

where

$$
\begin{aligned}
& \mathscr{R} \stackrel{\text { def }}{=}\left(\rho^{2} \mathscr{A}^{\prime}\right)^{\prime} u_{x}^{2}-2 \rho \mathscr{A}^{\prime}\left[u_{t}+u u_{x}+\varpi_{x}\right]_{x}+\left(\rho \mathscr{V}^{\prime \prime} / \mathscr{A}^{\prime}\right)^{\prime} \mathscr{A}_{x}^{2}, \\
& m \stackrel{\text { def }}{=} \rho u-2 \epsilon\left[\rho \mathscr{A}^{\prime} u_{x}\right]_{x} .
\end{aligned}
$$

Introducing the linear Sturm-Liouville operator $\mathcal{L}_{\rho} \stackrel{\text { def }}{=} \rho-2 \epsilon \partial_{x} \rho \mathscr{A}^{\prime} \partial_{x}$, the equation (48) multiplied by $\rho$ becomes

$$
\mathcal{L}_{\rho}\left\{u_{t}+u u_{x}+\varpi_{x}\right\}+\epsilon\left[\left(\rho^{2} \mathscr{A}^{\prime}\right)^{\prime} u_{x}^{2}+\left(\rho^{\prime \prime} / \mathscr{A}^{\prime}\right)^{\prime} \mathscr{A}_{x}^{2}\right]_{x}=0
$$

or, inverting the operator,

$$
u_{t}+u u_{x}+\varpi_{x}=-\epsilon \mathcal{G}_{\rho} \partial_{x}\left\{\left(\rho^{2} \mathscr{A}^{\prime}\right)^{\prime} u_{x}^{2}+\left(\rho^{\prime \prime} / \mathscr{A}^{\prime}\right)^{\prime} \mathscr{A}_{x}^{2}\right\},
$$

where $\mathcal{G}_{\rho}=\mathcal{L}_{\rho}^{-1}$. The operator $\mathcal{G}_{\rho} \partial_{x}$ acting on high frequencies like a first-order antiderivative, it has a smoothing effect. However, this equation is in a non-conservative form. A conservative variant is obtained multiplying (55) by $\rho$ and exploiting the mass conservation, hence

$$
[\rho u]_{t}+\left[\rho u^{2}+\rho \mathscr{V}^{\prime}-\mathscr{V}+\epsilon \mathcal{J}_{\rho}\left\{\left(\rho^{2} \mathscr{A}^{\prime}\right)^{\prime} u_{x}^{2}+\left(\rho^{\prime \prime} / \mathscr{A}^{\prime}\right)^{\prime} \mathscr{A}_{x}^{2}\right\}\right]_{x}=0
$$

with the operator

$$
\begin{aligned}
\partial_{\rho} & \stackrel{\text { def }}{=} \partial_{x}^{-1} \rho \mathcal{G}_{\rho} \partial_{x}=\partial_{x}^{-1} \rho\left[1-2 \epsilon \rho^{-1} \partial_{x} \rho \mathscr{A}^{\prime} \partial_{x}\right]^{-1} \rho^{-1} \partial_{x} \\
& =\left[1-2 \epsilon \rho \mathscr{A}^{\prime} \partial_{x} \rho^{-1} \partial_{x}\right]^{-1}=1+2 \varepsilon \rho \mathscr{A}^{\prime} \partial_{x} \mathcal{G}_{\rho} \partial_{x} .
\end{aligned}
$$


Comparing (56) with (49), one obtains at once an alternative expression for the regularising term

$$
\mathscr{R}=\mathcal{J}_{\rho}\left\{\left(\rho^{2} \mathscr{A}^{\prime}\right)^{\prime} u_{x}^{2}+\left(\rho^{\prime \prime} / \mathscr{A}^{\prime}\right)^{\prime} \mathscr{A}_{x}^{2}\right\} .
$$

While the definition (52) of $\mathscr{R}$ involves second-order spacial derivatives, the alternative form (58) shows actually that $\mathscr{R}$ behaves at high frequencies somehow like zeroth-order derivatives. Moreover, since the relations (52) and (58) are identical, we obtain yet another form of the momentum equation

$$
2 \rho \mathscr{A}^{\prime}\left[u_{t}+u u_{x}+\varpi_{x}\right]_{x}+\left(\mathcal{J}_{\rho}-\mathcal{J}\right)\left\{\left(\rho^{2} \mathscr{A}^{\prime}\right)^{\prime} u_{x}^{2}+\left(\rho^{\prime \prime} / \mathscr{A}^{\prime}\right)^{\prime} \mathscr{A}_{x}^{2}\right\}=0,
$$

where $\mathcal{J}$ is the identity operator. Note that applying the operator $\partial_{x}^{-1}\left(2 \rho \mathscr{A}^{\prime}\right)^{-1}$, the equation (59) can be rewritten

$$
u_{t}+u u_{x}+\varpi_{x}+\partial_{x}^{-1}\left(2 \rho \mathscr{A}^{\prime}\right)^{-1}\left(\mathcal{J}-\mathcal{J}_{\rho}^{-1}\right)\{\mathscr{R}\}=0,
$$

and with

$$
\mathcal{J}-\mathcal{J}_{\rho}^{-1}=\mathcal{J}-\partial_{x}^{-1} \rho\left[1-2 \epsilon \rho^{-1} \partial_{x} \rho \mathscr{A}^{\prime} \partial_{x}\right] \rho^{-1} \partial_{x}=2 \epsilon \rho \mathscr{A}^{\prime} \partial_{x} \rho^{-1} \partial_{x},
$$

one gets the equation (48), as it should be.

3.5. Rankine-Hugoniot conditions. Here, we assume that $\rho_{x}$ and $u_{x}$ are both continuous if $\epsilon>0$ and that discontinuities (if any) occur only in $\rho_{x x}$ and $u_{x x}$. Differentiating twice with respect of $x$ the mass conservation (42), the jump condition of the resulting equation is

$$
(u-\dot{s}) \llbracket \rho_{x x} \rrbracket+\rho \llbracket u_{x x} \rrbracket=0,
$$

while the jump condition for (47) is (provided that $\epsilon$ and $\mathscr{A}^{\prime}$ are not zero)

$$
(u-\dot{s}) \llbracket u_{x x} \rrbracket+\mathscr{V}^{\prime \prime} \llbracket \rho_{x x} \rrbracket=0 .
$$

Thus, the speed of the regularised shock is identical to the original one, whatever the function $\mathscr{A}^{\prime} \neq 0$ is. Therefore, a suitable choice for the function $\mathscr{A}$ cannot be determined by this consideration.

3.6. Hamiltonian formulation. Introducing the momentum $m \stackrel{\text { def }}{=} \rho u-2 \epsilon\left[\rho \mathscr{A}^{\prime} u_{x}\right]_{x}$ and the Hamiltonian functional density

$$
\mathscr{H}_{\epsilon}(\rho, m) \stackrel{\text { def }}{=} \frac{1}{2} m \mathcal{G}_{\rho}\{m\}+\mathscr{V}+\epsilon \mathscr{A}^{\prime} \mathscr{V}^{\prime \prime} \rho_{x}^{2},
$$

we have

$$
\begin{aligned}
\mathcal{E}_{m}\left\{\mathscr{H}_{\epsilon}\right\} & =\mathcal{G}_{\rho}\{m\}=u, \\
\mathcal{E}_{\rho}\left\{\mathscr{H}_{\epsilon}\right\} & =\mathscr{V}^{\prime}-\epsilon\left(\mathscr{A}^{\prime} \mathscr{V}^{\prime \prime}\right)^{\prime} \rho_{x}^{2}-2 \epsilon \mathscr{A}^{\prime} \mathscr{V}^{\prime \prime} \rho_{x x}-\frac{1}{2} u^{2}-\epsilon\left(\rho \mathscr{A}^{\prime}\right)^{\prime} u_{x}^{2},
\end{aligned}
$$

where $\mathcal{E}_{m}$ and $\mathcal{E}_{\rho}$ are the Euler-Lagrange operators with respect of $m$ and $\rho$. The rbE equations have then the Hamiltonian structure

$$
\partial_{t}\left(\begin{array}{c}
\rho \\
m
\end{array}\right)=-\mathbb{J} \cdot\left(\begin{array}{c}
\mathcal{E}_{\rho}\left\{\mathscr{H}_{\epsilon}\right\} \\
\mathcal{E}_{m}\left\{\mathscr{H}_{\epsilon}\right\}
\end{array}\right), \quad \mathbb{J} \stackrel{\text { def }}{=}\left[\begin{array}{cc}
0 & \partial_{x} \rho \\
\rho \partial_{x} & m \partial_{x}+\partial_{x} m
\end{array}\right],
$$


yielding the equations (42) and (50). It should be noted that $\mathbb{J}$ being skew-symmetric and satisfying the Jacobi identity [43], it is a proper Hamiltonian (Lie-Poisson) operator.

3.7. Steady motions. We seek here for solutions independent of the time $t$, i.e., we look for travelling waves of permanent form observed in the frame of reference moving with the wave (note that the rbE equations are Galilean invariant). For such flows, the mass conversation yields

$$
u=I / \rho,
$$

where $I$ is an integration constant (the mean impulse). From the relations (49) and (51), the mean (constant) momentum and energy fluxes are respectively

$$
\begin{aligned}
& S=\rho u^{2}+\rho \mathscr{V}^{\prime}-\mathscr{V}+\epsilon \mathscr{R}, \\
& F=\left(\frac{1}{2} \rho u^{2}+\rho \mathscr{V}^{\prime}+\epsilon \rho \mathscr{A}^{\prime} u_{x}^{2}+\epsilon \mathscr{A}^{\prime} \mathscr{V}^{\prime \prime} \rho_{x}^{2}+\epsilon \mathscr{R}\right) u+2 \epsilon \rho \mathscr{A}^{\prime} \mathscr{V}^{\prime \prime} \rho_{x} u_{x},
\end{aligned}
$$

thence - eliminating $\mathscr{R}$ and using (68) — the ordinary differential equation

$$
\frac{2 \epsilon \mathscr{A}^{\prime}}{\rho^{2}}\left(\frac{\mathrm{d} \rho}{\mathrm{d} x}\right)^{2}=\frac{I^{2}-2 S \rho+2(F / I) \rho^{2}-2 \rho \mathscr{V}}{I^{2}-\rho^{3} \mathscr{V}^{\prime \prime}} .
$$

Considering equilibrium states in the far field - i.e., $\rho \rightarrow \rho_{ \pm}$and $u \rightarrow u_{ \pm}$as $x \rightarrow \pm \infty$, $\rho_{ \pm}$and $u_{ \pm}$being constants - we have $\mathscr{R} \rightarrow 0$ and the fluxes in the far field are

$$
I_{ \pm} \stackrel{\text { def }}{=} \rho_{ \pm} u_{ \pm}, \quad S_{ \pm} \stackrel{\text { def }}{=} \rho_{ \pm} u_{ \pm}^{2}+\rho_{ \pm} \mathscr{V}_{ \pm}^{\prime}-\mathscr{V}_{ \pm}, \quad F_{ \pm} \stackrel{\text { def }}{=} \frac{1}{2} \rho_{ \pm} u_{ \pm}^{3}+\rho_{ \pm} \mathscr{V}_{ \pm}^{\prime} u_{ \pm} .
$$

For regular solutions, the fluxes of mass, momentum and energy are constants, so $I_{+}=$ $I_{-}=I, S_{+}=S_{-}=S$ and $F_{+}=F_{-}=F$. For weak solutions, however, we assume that only the mass and momentum are conserved (i.e., $I_{+}=I_{-}=I$ and $S_{+}=S_{-}=S$ ), some energy being lost at the singularity (shock) so $F_{+} \neq F_{-}$.

It should be noted that $\mathscr{A}$ does not appear in the relations (72). The role of $\mathscr{A}$ is to control the singularity at the shock. So, a priori, a local analysis of a shock is necessary to obtain further informations on $\mathscr{A}$.

3.8. Local analysis of steady solution. Let assume that we have a (weak) steady solution with far field conditions (72) and with, possibly, only one singularity at $x=0$ where the density is assumed on the form

$$
\rho=\bar{\rho}+\varrho_{ \pm}|x|^{\alpha}+\mathrm{o}\left(|x|^{\alpha}\right)
$$

where $\alpha>0$ is a constant to be found. The plus and minus subscripts in $\varrho$ denote $x>0$ and $x<0$, respectively. With $f_{0} \stackrel{\text { def }}{=} f(\bar{\rho})$ for any function $f$, the constant mass flux (68) yields

$$
u=\frac{I}{\bar{\rho}}\left(1-\frac{\varrho_{ \pm}}{\bar{\rho}}|x|^{\alpha}\right)+\mathrm{o}\left(|x|^{\alpha}\right)
$$

thence

$$
\mathscr{R}=2 \alpha(\alpha-1) \varrho_{ \pm} \bar{\rho}^{-2} \mathscr{A}_{0}^{\prime}\left(I^{2}-\bar{\rho}^{3} \mathscr{V}_{0}^{\prime \prime}\right)|x|^{\alpha-2}+\mathrm{o}\left(|x|^{\alpha-2}\right)
$$


and the ODE (71) yields

$$
\frac{2 \epsilon \mathscr{A}_{0}^{\prime} \alpha^{2} \varrho_{ \pm}^{2}}{\bar{\rho}^{2}}|x|^{2 \alpha-2}+\mathrm{o}\left(|x|^{2 \alpha-2}\right)=\frac{I^{2}-2 S \bar{\rho}+2\left(F_{ \pm} / I\right) \bar{\rho}^{2}-2 \bar{\rho}^{\mathscr{V}}+\mathrm{O}\left(|x|^{\alpha}\right)}{I^{2}-\bar{\rho}^{3} \mathscr{V}_{0}^{\prime \prime}-\left(3 \bar{\rho}^{2} \mathscr{V}_{0}^{\prime \prime}+\bar{\rho}^{3} \mathscr{V}_{0}^{\prime \prime \prime}\right) \varrho_{ \pm}|x|^{\alpha}+\mathrm{o}\left(|x|^{\alpha}\right)} .
$$

From (75) there are three (necessary) possibilities to obtain admissible solutions: $\alpha=1$ or $\alpha>1$ or $I^{2}=\bar{\rho}^{3} \mathscr{V}_{0}^{\prime \prime}$.

If $I^{2} \neq \bar{\rho}^{3} \mathscr{V}_{0}^{\prime \prime}$, the expansions (73) and (74) substituted into (69) and (70) show that $S$ and $F$ cannot be constant. Therefore, there are no solutions behaving like (73) if $I^{2} \neq \bar{\rho}^{3} \mathscr{V}_{0}^{\prime \prime}$.

If $I^{2}=\bar{\rho}^{3} \mathscr{V}_{0}^{\prime \prime}$, the equation (76) implies that $\alpha=2 / 3$ if $\mathscr{V}_{0} \neq I^{2} / 2 \bar{\rho}-S+\left(F_{ \pm} / I\right) \bar{\rho}$ and $\alpha=1$ if $\mathscr{V}_{0}=I^{2} / 2 \bar{\rho}-S+\left(F_{ \pm} / I\right) \bar{\rho}$. The latter case does not yield constant $S$ and $F$, so it must be rejected. Finally, the only possibility is $\alpha=2 / 3$ and (76) gives

$$
\frac{8 \epsilon \mathscr{A}_{0}^{\prime} \varrho_{ \pm}^{2}}{9 \bar{\rho}^{3}}=-\frac{I^{2}-2 S \bar{\rho}+2\left(F_{ \pm} / I\right) \bar{\rho}^{2}-2 \bar{\rho} \mathscr{V}_{0}}{3 I^{2}+\bar{\rho}^{4} \mathscr{V}_{0}^{\prime \prime \prime}}
$$

In summary, the local analysis does not gives hints for a suitable choice of $\mathscr{A}$. However, as in [44], we found the interesting feature that stationary weak solutions have universal singularities as $|x|^{2 / 3}$, whatever the potential $\mathscr{V}$ is and for all possible regularising functions $\mathscr{A}$. Note that the analysis above does not rule out the possibility of different type of singularities such as $|x|^{\alpha}(\log |x|)^{\beta}$.

\section{LOCAL WELL-POSEDNESS OF THE REGULARISED BAROTROPIC EULER SYSTEM}

The aim of this section is to prove the local well-posedness of the regularised barotropic Euler system introduced in Section 3.

Let $P=P(\rho)$ denotes the pressure, and let $\rho=\tilde{\rho}+\bar{\rho}$, where $\bar{\rho}$ is a positive constant. Let also

$$
\varpi(\rho) \stackrel{\text { def }}{=} \int_{\bar{\rho}}^{\rho} \frac{P^{\prime}(\alpha)}{\alpha} \mathrm{d} \alpha, \quad \mathscr{V} \stackrel{\text { def }}{=} \int_{\bar{\rho}}^{\rho} \varpi(\alpha) \mathrm{d} \alpha,
$$

where the prime denotes the derivative with respect to $\rho$.

Recalling the operator $\mathcal{L}_{\rho} \stackrel{\text { def }}{=} \rho-2 \epsilon \partial_{x} \rho \mathscr{A}^{\prime} \partial_{x}$ and the system (42), (55)

$$
\begin{aligned}
\rho_{t}+[\rho u]_{x} & =0, \\
u_{t}+u u_{x}+\varpi_{x} & =-\epsilon \mathcal{L}_{\rho}^{-1} \partial_{x}\left\{\left(\rho^{2} \mathscr{A}^{\prime}\right)^{\prime} u_{x}^{2}+\left(\rho^{\prime \prime} / \mathscr{A}^{\prime}\right)^{\prime} \mathscr{A}^{\prime 2} \rho_{x}^{2}\right\},
\end{aligned}
$$

where smooth solutions of (79), (80) satisfy the energy equation (51) with $\mathscr{R}$ is defined in (58). The goal of this section is to prove the following theorem

Theorem 1. Let $\tilde{m} \geqslant s \geqslant 2, \tilde{m}$ be an integer, $P, \mathscr{A} \in C^{\tilde{m}+4}(] 0,+\infty[)$ such that $P^{\prime}(\rho)>0$, $\mathscr{A}^{\prime}(\rho)>0$ for $\rho>0$. Let also $W_{0}=\left(\tilde{\rho}_{0}, u_{0}\right)^{\top} \in H^{s}$ satisfying $\inf _{x \in \mathbb{R}} \rho_{0}(x)>\rho^{*}$, then there exist $T>0$ and a unique solution $W \in C\left([0, T], H^{s}\right) \cap C^{1}\left([0, T], H^{s-1}\right)$ of (79), (80) satisfying the non-emptiness condition $\inf _{x \in \mathbb{R}} \rho(t, x)>0$, and the conservation of the energy

$$
\frac{\mathrm{d}}{\mathrm{d} t} \int_{\mathbb{R}}\left(\frac{1}{2} \rho u^{2}+\epsilon \rho \mathscr{A}^{\prime} u_{x}^{2}+\mathscr{V}+\epsilon \mathscr{A}^{\prime} \mathscr{V}^{\prime \prime} \rho_{x}^{2}\right) \mathrm{d} x=0
$$


Moreover, if the maximal existence time $T_{\max }<+\infty$, then

$$
\lim _{t \rightarrow T_{\max }}\left\|W_{x}\right\|_{L^{\infty}}=+\infty .
$$

Remark 1. The solution given in the previous theorem depends continuously on the initial data in the sense: If $W_{0}, \tilde{W}_{0} \in H^{s}$, such that $\rho_{0}, \tilde{\rho}_{0} \geqslant \rho^{*}$, then there exists a constant $C\left(\|\tilde{W}\|_{L^{\infty}\left([0, T], H^{s}\right)},\|W\|_{L^{\infty}\left([0, T], H^{s}\right)}\right)>0$, such that

$$
\|W-\tilde{W}\|_{L^{\infty}\left([0, T], H^{s-1}\right)} \leqslant C\left\|W_{0}-\tilde{W}_{0}\right\|_{H^{s}} .
$$

Remark 2. Theorem 1 holds also for periodic domains.

Remark 3. Note that if $\left.\rho \in\left[\rho_{\text {inf }}, \rho_{\text {sup }}\right] \subset\right] 0,+\infty\left[\right.$, then $0<\alpha \leqslant P^{\prime}(\rho) / \rho \leqslant \beta<+\infty$. This implies with the definition (78) that $\alpha(\rho-\bar{\rho})^{2} \leqslant \mathscr{V} \leqslant \beta(\rho-\bar{\rho})^{2}$. Then, the conserved energy (81) is equivalent to the $H^{1}$ norm of $(\tilde{\rho}, u)$.

4.1. Preliminary results. Let $\Lambda$ be defined such that $\widehat{\Lambda f}=\left(1+\xi^{2}\right)^{\frac{1}{2}} \hat{f}$. In order to prove Theorem 1, we recall the classical lemmas.

Lemma 1. ([29]) Let $[A, B] \stackrel{\text { def }}{=} A B-B A$ be the commutator of the operators $A$ and $B$. If $r \geqslant 0$, then

$$
\begin{aligned}
\|f g\|_{H^{r}} & \lesssim\|f\|_{L^{\infty}}\|g\|_{H^{r}}+\|f\|_{H^{r}}\|g\|_{L^{\infty}}, \\
\left\|\left[\Lambda^{r}, f\right] g\right\|_{L^{2}} & \lesssim\left\|f_{x}\right\|_{L^{\infty}}\|g\|_{H^{r-1}}+\|f\|_{H^{r}}\|g\|_{L^{\infty}} .
\end{aligned}
$$

Lemma 2. ([15]) Let $F \in C^{\tilde{m}+2}(\mathbb{R})$ with $F(0)=0$ and $0 \leqslant s \leqslant \tilde{m}$, then there exists a continuous function $\tilde{F}$, such that for all $f \in H^{s} \cap W^{1, \infty}$ we have

$$
\|F(f)\|_{H^{s}} \leqslant \tilde{F}\left(\|f\|_{W^{1, \infty}}\right)\|f\|_{H^{s}} .
$$

In the following lemma, we prove the invertibility of the operator $\mathcal{L}_{\rho}(4)$ and we obtain some estimates satisfied by $\mathcal{L}_{\rho}^{-1}$.

Lemma 3. Let $0<\rho_{\text {inf }} \leqslant \rho \in W^{1, \infty}$ and $\mathscr{A} \in C^{2}(] 0,+\infty[)$ satisfying $\mathscr{A}^{\prime}>0$, then the operator $\mathcal{L}_{\rho}$ is an isomorphism from $H^{2}$ to $L^{2}$ and

(1) If $0 \leqslant s \leqslant \tilde{m} \in \mathbb{N}$ and $\mathscr{A} \in C^{\tilde{m}+3}(] 0,+\infty[)$, then

$$
\begin{aligned}
\left\|\mathcal{L}_{\rho}^{-1} \partial_{x} \psi\right\|_{H^{s+1}} & \lesssim\|\psi\|_{H^{s}}+\|\rho-\bar{\rho}\|_{H^{s}}\left\|\mathcal{L}_{\rho}^{-1} \partial_{x} \psi\right\|_{W^{1, \infty}} \\
\left\|\mathcal{L}_{\rho}^{-1} \phi\right\|_{H^{s+1}} & \lesssim\|\phi\|_{H^{s}}+\|\rho-\bar{\rho}\|_{H^{s}}\left\|\mathcal{L}_{\rho}^{-1} \phi\right\|_{W^{1, \infty}} .
\end{aligned}
$$

(2) If $0 \leqslant s \leqslant \tilde{m} \in \mathbb{N}$ and $\mathscr{A} \in C^{\tilde{m}+3}(] 0,+\infty[)$, then

$$
\begin{aligned}
\left\|\mathcal{L}_{\rho}^{-1} \partial_{x} \psi\right\|_{H^{s+1}} & \lesssim\|\psi\|_{H^{s}}\left(1+\|\rho-\bar{\rho}\|_{H^{s}}\right), \\
\left\|\mathcal{L}_{\rho}^{-1} \phi\right\|_{H^{s+1}} & \lesssim\|\phi\|_{H^{s}}\left(1+\|\rho-\bar{\rho}\|_{H^{s}}\right),
\end{aligned}
$$

(3) If $\phi \in C_{\lim } \stackrel{\text { def }}{=}\{f \in C, f( \pm \infty) \in \mathbb{R}\}$, then $\mathcal{L}_{\rho}^{-1} \phi$ is well defined and

$$
\left\|\mathcal{L}_{\rho}^{-1} \phi\right\|_{W^{2, \infty}} \lesssim\|\phi\|_{L^{\infty}} .
$$


(4) If $\psi \in C_{\lim } \cap L^{1}$, then

$$
\left\|\mathcal{L}_{\rho}^{-1} \partial_{x} \psi\right\|_{W^{1, \infty}} \lesssim\|\psi\|_{L^{\infty}}+\|\psi\|_{L^{1}} .
$$

All the constants depend on $s, \varepsilon, \rho_{\mathrm{inf}},\|\rho-\bar{\rho}\|_{W^{1, \infty}}$ and not on $\|\rho-\bar{\rho}\|_{H^{s}}$.

The previous lemma is proven in [36] for the special case $\mathscr{A}(\rho)=\rho^{3} / 6$. Here, the same proof is followed

Proof. Step 0: In the first step, we prove, using the Lax-Milgram theorem, that $\mathcal{L}_{\rho}$ is an isomorphism from $H^{2}$ to $L^{2}$, let the bi-linear function $a$ from $H^{1} \times H^{1}$ to $\mathbb{R}$ such that

$$
a(u, v) \stackrel{\text { def }}{=}(\rho u, v)+2 \varepsilon\left(\rho \mathscr{A}^{\prime} u_{x}, v_{x}\right) .
$$

Using that $\rho$ is bounded and far from zero, one can easily show that the function $a$ is continuous and coercive, then Lax-Milgram theorem shows that there exists a continuous bijection $J$ between $H^{1}$ and $H^{-1}$, such that for all $u, v \in H^{1}$ we have

$$
a(u, v)=(J u, v)_{H^{-1} \times H^{1}} .
$$

If $J u \in L^{2}$, and integration by parts shows that $2 \varepsilon\left(\rho \mathscr{A}^{\prime} u_{x}\right)_{x}=\rho u-J u \in L^{2}$ and $J=\mathcal{L}_{\rho}$, this implies that $u \in H^{2}$ which finishes the proof that $\mathcal{L}_{\rho}$ is an isomorphism from $H^{2}$ to $L^{2}$.

Step 1: Let $\mathcal{L}_{\rho} u=\phi+\psi_{x}$, then

$$
\begin{aligned}
\|u\|_{H^{1}}^{2} & =(u, u)+\left(u_{x}, u_{x}\right) \\
& \lesssim(\rho u, u)+2 \varepsilon\left(\rho \mathscr{A}^{\prime} u_{x}, u_{x}\right) \\
& =\left(\mathcal{L}_{\rho} u, u\right)=(\phi, u)-\left(\psi, u_{x}\right) \\
& \lesssim\|u\|_{H^{1}}\left(\|\phi\|_{L^{2}}+\|\psi\|_{L^{2}}\right),
\end{aligned}
$$

which implies that

$$
\|u\|_{H^{1}} \lesssim\|\phi\|_{L^{2}}+\|\psi\|_{L^{2}}
$$

Using the Young inequality $a b \leqslant \frac{1}{2 \alpha} a^{2}+\frac{\alpha}{2} b^{2}$ with $\alpha>0$ we obtain

$$
\begin{aligned}
\left\|u_{x}\right\|_{H^{1}}^{2} & =\left(u_{x}, u_{x}\right)+\left(u_{x x}, u_{x x}\right) \\
& \lesssim\left(\rho u_{x}, u_{x}\right)+2 \varepsilon\left(\rho \mathscr{A}^{\prime} u_{x x}, u_{x x}\right) \\
& =-\left(\rho u, u_{x x}\right)-\left(\rho_{x} u, u_{x}\right)+2 \varepsilon\left(\left(\rho \mathscr{A}^{\prime} u_{x}\right)_{x}-\left(\rho \mathscr{A}^{\prime}\right)_{x} u_{x}, u_{x x}\right) \\
& =-\left(\mathcal{L}_{\rho} u, u_{x x}\right)-\left(\rho_{x} u, u_{x}\right)-2 \varepsilon\left(\left(\rho \mathscr{A}^{\prime}\right)_{x} u_{x}, u_{x x}\right) \\
& \lesssim \alpha\left\|u_{x x}\right\|_{L^{2}}^{2}+\frac{1}{\alpha}\left(\left\|\mathcal{L}_{\rho} u\right\|_{L^{2}}^{2}+\left\|u_{x}\right\|_{L^{2}}^{2}\right)+\|u\|_{H^{1}}^{2} .
\end{aligned}
$$

Taking $\alpha>0$ small enough we obtain that

$$
\left\|u_{x}\right\|_{H^{1}}^{2} \lesssim\left\|\mathcal{L}_{\rho} u\right\|_{L^{2}}^{2}+\|u\|_{H^{1}}^{2} .
$$

then

$$
\left\|u_{x}\right\|_{H^{1}} \lesssim\left\|\mathcal{L}_{\rho} u\right\|_{L^{2}}+\|u\|_{H^{1}} .
$$

Taking $\phi=0$ (respectively $\psi=0$ ) and using (91), we obtain

$$
\left\|\mathcal{L}_{\rho}^{-1} \partial_{x} \psi\right\|_{H^{1}} \lesssim\|\psi\|_{H^{1}} \quad\left\|\mathcal{L}_{\rho}^{-1} \phi\right\|_{H^{s+1}} \lesssim\|\phi\|_{L^{2}}
$$


An interpolation with (91) implies that

$$
\left\|\mathcal{L}_{\rho}^{-1} \partial_{x} \psi\right\|_{H^{s+1}} \lesssim\|\psi\|_{H^{s}}, \quad\left\|\mathcal{L}_{\rho}^{-1} \phi\right\|_{H^{s+1}} \lesssim\|\phi\|_{H^{s}} \quad \forall s \in[0,1] .
$$

Let now $s>0$, and let $\mathcal{L}_{\rho} u=\phi+\psi_{x}$, we then have

$$
\mathcal{L}_{\rho} \Lambda^{s} u=\left[\rho, \Lambda^{s}\right] u+\Lambda^{s} \phi+\partial_{x}\left\{-2 \varepsilon\left[\rho \mathscr{A}^{\prime}, \Lambda^{s}\right] u_{x}+\Lambda^{s} \psi\right\} .
$$

Defining $\tilde{u}=\Lambda^{s} u, \tilde{\phi}=\left[\rho, \Lambda^{s}\right] u+\Lambda^{s} \phi$ and $\tilde{\psi}=-2 \varepsilon\left[\rho \mathscr{A}^{\prime}, \Lambda^{s}\right] u_{x}+\Lambda^{s} \psi$ and using (91), (85), (86) we obtain

$$
\begin{aligned}
\left\|\Lambda^{s} u\right\|_{H^{1}} & \lesssim\left\|\left[\rho, \Lambda^{s}\right] u\right\|_{L^{2}}+\left\|\left[\rho \mathscr{A}^{\prime}, \Lambda^{s}\right] u_{x}\right\|_{L^{2}}+\|\phi\|_{H^{s}}+\|\psi\|_{H^{s}} \\
& \lesssim\left\|\Lambda^{s-1} u\right\|_{H^{1}}+\|\rho-\bar{\rho}\|_{H^{s}}\|u\|_{W^{1, \infty}}+\|\phi\|_{H^{s}}+\|\psi\|_{H^{s}} .
\end{aligned}
$$

Then, by induction (on $s$ ) and using (92) one obtains that (87) holds for all $s \geqslant 0$.

Step 2: If $s \leqslant 1$, then (88) follows directly from (92). If $s>1$, using the embedding $H^{1} \hookrightarrow L^{\infty}$, (87) and (92) for $s=1$ we obtain (88).

Step 3: Let $C_{0} \stackrel{\text { def }}{=}\{f \in C, f( \pm \infty)=0\}$, using that $L^{2} \cap C_{0}$ is dense in $C_{0}$ one can define $\mathcal{L}_{\rho}^{-1}$ on $C_{0}$. If $\phi$ is in $C_{\text {lim }}$, we use the change of functions (see Lemma 4.4 in [36])

$$
\phi_{0}(x) \stackrel{\text { def }}{=} \phi(x)-\frac{1}{\bar{\rho}} \mathcal{L}_{\rho}\left(\phi(-\infty)+(\phi(+\infty)-\phi(-\infty)) \frac{\mathrm{e}^{x}}{1+\mathrm{e}^{x}}\right) \in C_{0},
$$

the operator $\mathcal{L}_{\rho}^{-1}$ can be defined as

$$
\mathcal{L}_{\rho}^{-1} \phi \stackrel{\text { def }}{=} \mathcal{L}_{\rho}^{-1} \phi_{0}+\frac{1}{\bar{\rho}}\left(\phi(-\infty)+(\phi(+\infty)-\phi(-\infty)) \frac{\mathrm{e}^{x}}{1+\mathrm{e}^{x}}\right) .
$$

In order order to prove (89), let $\phi=\mathcal{L}_{\rho} u$, using the variable

$$
z \stackrel{\text { def }}{=} \int \frac{\mathrm{d} x}{2 \rho(x) \mathscr{A}^{\prime}(\rho(x))},
$$

we obtain that

$$
\phi=\rho u-\frac{\varepsilon}{2 \rho \mathscr{A}^{\prime}} u_{z z} .
$$

The classical maximum principle equations implies that $\|u\|_{L^{\infty}} \leqslant\|\phi\|_{L^{\infty}} / \rho_{\text {inf }}$, which implies with (95) that $\left\|u_{z z}\right\|_{L^{\infty}} \lesssim\|\phi\|_{L^{\infty}}$, then the Landau-Kolmogorov inequality (see Lemma 4.3 in [36] for example) implies that $\left\|u_{z}\right\|_{L^{\infty}} \lesssim\|\phi\|_{L^{\infty}}$. The last inequality with the change of variables (94) imply that $\left\|u_{x}\right\|_{L^{\infty}} \lesssim\|\phi\|_{L^{\infty}}$, using that $2 \varepsilon \rho \mathscr{A}^{\prime} u_{x x}=\rho u-2 \varepsilon\left(\rho \mathscr{A}^{\prime}\right)_{x} u_{x}-\phi$ we obtain (89).

Step 4: Note that

$$
\mathcal{L}_{\rho} \int_{-\infty}^{x} \frac{\psi}{\rho \mathscr{A}^{\prime}} \mathrm{d} y=\rho \int_{-\infty}^{x} \frac{\psi}{\rho \mathscr{A}^{\prime}} \mathrm{d} y-2 \varepsilon \psi_{x}
$$


Applying $\mathcal{L}_{\rho}^{-1}$ and $\partial_{x}$ one obtains

$$
\begin{aligned}
2 \varepsilon \mathcal{L}_{\rho}^{-1} \partial_{x} \psi & =\mathcal{L}_{\rho}^{-1}\left(\rho \int_{-\infty}^{x} \frac{\psi}{\rho \mathscr{A}^{\prime}} \mathrm{d} y\right)-\int_{-\infty}^{x} \frac{\psi}{\rho \mathscr{A}^{\prime}} \mathrm{d} y \\
2 \varepsilon \partial_{x} \mathcal{L}_{\rho}^{-1} \partial_{x} \psi & =\partial_{x} \mathcal{L}_{\rho}^{-1}\left(\rho \int_{-\infty}^{x} \frac{\psi}{\rho \mathscr{A}^{\prime}} \mathrm{d} y\right)-\frac{\psi}{\rho \mathscr{A}^{\prime}} .
\end{aligned}
$$

The last two inequalities with (89) imply (90).

4.2. Iteration scheme and energy estimate. Defining $W \stackrel{\text { def }}{=}(\tilde{\rho}, u)^{\top}$ and

$$
B(W) \stackrel{\text { def }}{=}\left(\begin{array}{cc}
u & \rho \\
\varpi^{\prime} & u
\end{array}\right), \quad F(W) \stackrel{\text { def }}{=}\left(\begin{array}{c}
0 \\
-\epsilon \mathcal{L}_{\rho}^{-1} \partial_{x}\left\{\left(\rho^{2} \mathscr{A}^{\prime}\right)^{\prime} u_{x}^{2}+\left(\rho^{\prime \prime} / \mathscr{A}^{\prime}\right)^{\prime} \mathscr{A}^{\prime 2} \rho_{x}^{2}\right\}
\end{array}\right),
$$

the system (79), (80) becomes

$$
W_{t}+B(W) W_{x}=F(W), \quad W(0, x)=W_{0}(x) .
$$

The proof of the local existence of (96) is based on solving the linear hyperbolic system

$$
\partial_{t} W^{n+1}+B\left(W^{n}\right) \partial_{x} W^{n+1}=F\left(W^{n}\right), \quad W^{n}(0, x)=\left(\tilde{\rho}_{0}(x), u_{0}(x)\right)^{\top},
$$

for all $n \geqslant 0$, where $W^{0}(t, x)=\left(\tilde{\rho}_{0}(x), u_{0}(x)\right)^{\top}$. Then, uniform (on $n$ ) estimates of an energy that is equivalent to the $H^{s}$ norm will be given. Taking the limit $n \rightarrow \infty$, we obtain a solution of (96). Since $\varpi^{\prime}>0$, the system (97) is hyperbolic; which is an important point to solve each iteration in (97).

For the sake of simplicity, let be $\underline{W}=W^{n}$ (known on every step of the iteration) and let $W=W^{n+1}$ be the solution of the linear system

$$
W_{t}+B(\underline{W}) \partial_{x} W_{x}=F(\underline{W}), \quad W(0, x)=\left(\tilde{\rho}_{0}(x), u_{0}(x)\right)^{\top} .
$$

Note that a symmetriser of $\underline{B}=B(\underline{W})$ is

$$
\underline{A}=A(\underline{W}) \stackrel{\text { def }}{=}\left(\begin{array}{cc}
\varpi^{\prime} & 0 \\
0 & \rho
\end{array}\right) \text {. }
$$

Let the energy of (98) be defined as

$$
E(W) \stackrel{\text { def }}{=}\left(\Lambda^{s} W, \underline{A} \Lambda^{s} W\right),
$$

where $(\cdot, \cdot)$ is the scalar product in $L^{2}$. Since the matrix $\underline{A} \underline{B}$ is symmetric, a helpful feature for the energy estimates below, it justifies the use of $\underline{A}$ in the definition of the energy $E(W)$. Note that if $\rho$ is bounded and far from zero, then $E(W)$ is equivalent to $\|W\|_{H^{s}}^{2}$. In order to prove Theorem 1 , the following energy estimate is needed.

Theorem 2. Let $W=(\tilde{\rho}, u)^{\top}, \rho=\tilde{\rho}+\tilde{\rho}, s \geqslant 2$ and $\rho^{*}, R>0$ then there exist $K, T>0$ such that: if the initial data $\left(\tilde{\rho}_{0}, u_{0}\right) \in H^{s}$ satisfy

$$
\inf _{x \in \mathbb{R}} \rho_{0}(x)>\rho^{*}, \quad E\left(W_{0}\right)<R,
$$

and $\underline{W} \in C\left([0, T], H^{s}\right) \cap C^{1}\left([0, T], H^{s-1}\right)$, satisfying for all $t \in[0, T]$

$$
\underline{\rho} \geqslant \rho^{*}, \quad\left\|\underline{W}_{t}\right\|_{H^{s-1}} \leqslant K, \quad E(\underline{W}) \leqslant R,
$$


then there exists a unique $W \in C\left([0, T], H^{s}\right) \cap C^{1}\left([0, T], H^{s-1}\right)$ a solution of (98) such that

$$
\rho \geqslant \rho^{*}, \quad\left\|W_{t}\right\|_{H^{s-1}} \leqslant K, \quad E(W) \leqslant R .
$$

Proof. For the existence of $W$ see Appendix A in [27]. Defining $\underline{F} \stackrel{\stackrel{\text { def }}{=}}{F(} \underline{W})$ and using (98) we obtain

$$
\begin{aligned}
E(W)_{t}= & 2\left(\Lambda^{s} W_{t}, \underline{A} \Lambda^{s} W\right)+\left(\Lambda^{s} W, \underline{A}_{t} \Lambda^{s} W\right) \\
= & -2\left(\Lambda^{s} \underline{B} W_{x}, \underline{A} \Lambda^{s} W\right)-2\left(\Lambda^{s} \underline{F}, \underline{A} \Lambda^{s} W\right)+\left(\Lambda^{s} W, \underline{A}_{t} \Lambda^{s} W\right) \\
= & -2\left(\left[\Lambda^{s}, \underline{B}\right] W_{x}, \underline{A} \Lambda^{s} W\right)-2\left(\underline{B} \Lambda^{s} W_{x}, \underline{A} \Lambda^{s} W\right) \\
& -2\left(\Lambda^{s} \underline{F}, \underline{A} \Lambda^{s} W\right)+\left(\Lambda^{s} W, \underline{A}_{t} \Lambda^{s} W\right) \\
= & : I+I I+I I I+I V,
\end{aligned}
$$

Now, some bounds of the four terms will be given. Note that

$$
\begin{aligned}
-\frac{1}{2} I= & \left(\left[\Lambda^{s}, \underline{u}\right] \tilde{\rho}_{x}, \underline{\varpi^{\prime}} \Lambda^{s} \tilde{\rho}\right)+\left(\left[\Lambda^{s}, \underline{\rho}\right] u_{x}, \underline{\varpi^{\prime}} \Lambda^{s} \tilde{\rho}\right)+\left(\left[\Lambda^{s}, \underline{u}\right] u_{x}, \underline{\rho} \Lambda^{s} u\right) \\
& +\left(\left[\Lambda^{s}, \underline{\varpi^{\prime}}\right] \tilde{\rho}_{x}, \underline{\rho} \Lambda^{s} u\right) .
\end{aligned}
$$

Using (85) and (86) we obtain

$$
\begin{aligned}
\left|\left(\left[\Lambda^{s}, \underline{u}\right] \tilde{\rho}_{x}, \underline{\varpi^{\prime}} \Lambda^{s} \tilde{\rho}\right)\right| & \leqslant\left\|\left[\Lambda^{s}, \underline{u}\right] \tilde{\rho}_{x}\right\|_{L^{2}}\left\|\underline{\varpi^{\prime}} \Lambda^{s} \tilde{\rho}\right\|_{L^{2}} \\
& \lesssim\left(\|\underline{u}\|_{L^{\infty}}\left\|\tilde{\rho}_{x}\right\|_{H^{s-1}}+\|\underline{u}\|_{H^{s}}\left\|\tilde{\rho}_{x}\right\|_{L^{\infty}}\right)\left\|\underline{\varpi^{\prime}}\right\|_{L^{\infty}}\left\|\Lambda^{s} \tilde{\rho}\right\|_{L^{2}} \\
& \lesssim\|W\|_{H^{s}}^{2} \lesssim E(W) .
\end{aligned}
$$

All the terms of $I$ can be studied by the same way to obtain

$$
|I| \lesssim E(W)
$$

Using that $\underline{A}$ and $\underline{A B}$ are symmetric, an integration by parts yield to

$$
\left.|I I|=\left|\left(\Lambda^{s} W,(\underline{A B})_{x} \Lambda^{s} W\right)\right| \leqslant \| \underline{A B}\right)_{x}\left\|_{L^{\infty}}\right\| W\left\|_{H^{s}} \lesssim\right\| W \|_{H^{s}} \lesssim E(W) .
$$

Using the Young inequality $2 a b \leqslant a^{2}+b^{2}$ one obtains

$$
|I I I| \leqslant\|\underline{A}\|_{L^{\infty}}\left(\|\underline{F}\|_{H^{s}}^{2}+\|W\|_{H^{s}}^{2}\right)
$$

From the inequality (88) we have

$$
\|\underline{F}\|_{H^{s}} \lesssim\left\|\left(\underline{\rho}^{2} \underline{\mathscr{A}}^{\prime}\right)^{\prime} \underline{u}_{x}^{2}+\left(\underline{\rho} \underline{\mathscr{V}}^{\prime \prime} / \underline{\mathscr{A}}^{\prime}\right)^{\prime} \underline{\mathscr{A}}^{2} \underline{\rho}_{x}^{2}\right\|_{H^{s-1}},
$$

which implies with (84) and (86) that $\|\underline{F}\|_{H^{s}}$ is bounded, then

$$
|I I I| \lesssim E(W)+1
$$

Note that $\left\|\tilde{\rho}_{t}\right\|_{L^{\infty}} \leqslant\left\|\tilde{\tilde{\rho}}_{t}\right\|_{H^{s-1}} \leqslant K$, then

$$
|I V| \leqslant\left\|\underline{A}_{t}\right\|_{L^{\infty}}\|W\|_{H^{s}}^{2} \lesssim K E(W)
$$


The system (98) implies

$$
\begin{aligned}
\left\|W_{t}\right\|_{H^{s-1}} & =\left\|B(\underline{W}) W_{x}+\underline{F}\right\|_{H^{s-1}} \\
& \lesssim\|B(\underline{W})\|_{L^{\infty}}\left\|W_{x}\right\|_{H^{s-1}}+\|B(\underline{W})\|_{H^{s-1}}\left\|W_{x}\right\|_{L^{\infty}}+\|\underline{F}\|_{H^{s}} \\
& \lesssim E(W)+1 .
\end{aligned}
$$

All the constants hidden in "¿" do not depend on $K$ and $W$. Using (105), (106), (107) and (108) we obtain that

$$
\partial_{t} E(W) \leqslant C(K+1)[E(W)+1],
$$

which implies with Gronwall lemma that

$$
E(W) \leqslant\left[E\left(W_{0}\right)+1\right] \mathrm{e}^{C(K+1) t}-1 .
$$

Since $E\left(W_{0}\right)<R$, choosing first $K>0$ and then $T>0$ such that

$$
C(R+1) \leqslant K, \quad\left[E\left(W_{0}\right)+1\right] \mathrm{e}^{C(K+1) T}-1 \leqslant R
$$

we obtain with (109) and (111) that $\left\|W_{t}\right\|_{H^{s-1}} \leqslant K$ and $E(W) \leqslant R$. Since $\left\|\rho_{t}\right\|_{L^{\infty}} \lesssim$ $\|W\|_{H^{s-1}} \leqslant K$ and $\rho_{0}>\rho^{*}$ then taking $T$ small enough we have $\rho \geqslant \rho^{*}$.

4.3. Proof of Theorem 1. Theorem 2 shows that if the initial data satisfy (101), then the sequence $\left(W^{n}\right)_{n \in \mathbb{N}}$ exists, it is uniformly bounded in $C\left([0, T], H^{s}\right) \cap C^{1}\left([0, T], H^{s-1}\right)$ and satisfies $\rho^{n} \geqslant \rho^{*}$. Using classical arguments of Sobolev spaces one can prove that there exists $W \in C\left([0, T], H^{s}\right)$ such that $W^{n}$ converges "up to a sub-sequence" to $W$ in $C\left([0, T], H^{s^{\prime}}\right)$ for all $s^{\prime} \in[0, s[$. Before taking the limit $n \rightarrow \infty$ in (97), we will verify that if $W^{n}$ converges, then $W^{n+1}$ converges too and towards the same limit. For that purpose, let

$$
\tilde{E}^{n} \stackrel{\text { def }}{=}\left(\Lambda^{s-1}\left(W^{n+1}-W^{n}\right), A^{n} \Lambda^{s-1}\left(W^{n+1}-W^{n}\right)\right),
$$

using estimates as in the proof of Theorem 2 (see also [39, 44] for more details) one can prove that for $T>0$ small enough, we obtain that $\tilde{E}^{n+1} \leqslant \tilde{E}^{n} / 2$, which implies that $\left\|W^{n+1}-W^{n}\right\|_{H^{s-1}} \rightarrow 0$. Taking $n \rightarrow \infty$ in the weak formulation of (97), we obtain that $W$ is a weak solution of (96). Using that $W \in C\left([0, T], H^{s}\right)$ and (96) we deduce that $W$ is a strong solution and $W \in C^{1}\left([0, T], H^{s-1}\right)$.

In order to prove the blow-up criteria (82), we suppose that $\left\|W_{x}\right\|_{L^{\infty}}$ is bounded and we prove that $\rho$ is far from zero and $\|W\|_{H^{s}}$ is bounded on any bounded time interval $[0, T]$. Using the characteristics

$$
\chi(0, x)=x, \quad \chi_{t}(t, x)=u(t, \chi(t, x)),
$$

the conservation of the mass (79) becomes

$$
\frac{\mathrm{d}}{\mathrm{d} t} \rho+u_{x} \rho=0, \quad \Longrightarrow \quad \rho_{0}(x) \mathrm{e}^{-t\left\|u_{x}\right\|_{L^{\infty}}} \leqslant \rho(t, x) \leqslant \rho_{0}(x) \mathrm{e}^{t\left\|u_{x}\right\|_{L^{\infty}}},
$$

which implies that $\rho$ is bounded and far from zero. The conservation of the energy (81) with the Sobolev embedding $H^{1} \hookrightarrow L^{\infty}$ imply that $\|W\|_{L^{\infty}}$ is bounded. 
Now, we will use that $\rho$ is far from zero and the boundness of $\|W\|_{W^{1, \infty}}$ to prove that $\|W\|_{H^{s}}$ is also bounded. As in the proof of Theorem 2, let

$$
\begin{gathered}
A(W) \stackrel{\text { def }}{=}\left(\begin{array}{cc}
\varpi^{\prime} & 0 \\
0 & \rho
\end{array}\right), \quad B(W) \stackrel{\text { def }}{=}\left(\begin{array}{cc}
u & \rho \\
\varpi^{\prime} & u
\end{array}\right), \quad \tilde{E}(W) \stackrel{\text { def }}{=}\left(\Lambda^{s} W, A \Lambda^{s} W\right), \\
F(W) \stackrel{\text { def }}{=}\left(\begin{array}{c}
0 \\
-\epsilon \mathcal{L}_{\rho}^{-1} \partial_{x}\left\{\left(\rho^{2} \mathscr{A}^{\prime}\right)^{\prime} u_{x}^{2}+\left(\rho \mathscr{V}^{\prime \prime} / \mathscr{A}^{\prime}\right)^{\prime} \mathscr{A}^{\prime 2} \rho_{x}^{2}\right\}
\end{array}\right),
\end{gathered}
$$

the system (79), (80) then becomes

$$
W_{t}+B(W) W_{x}=F(W) .
$$

As in (104), we have

$$
\begin{aligned}
\tilde{E}(W)_{t}= & -2\left(\left[\Lambda^{s}, B\right] W_{x}, A \Lambda^{s} W\right)-2\left(B \Lambda^{s} W_{x}, A \Lambda^{s} W\right) \\
& -2\left(\Lambda^{s} F, A \Lambda^{s} W\right)+\left(\Lambda^{s} W, A_{t} \Lambda^{s} W\right) \\
= & : I+I I+I I I+I V .
\end{aligned}
$$

Note that

$-\frac{1}{2} I=\left(\left[\Lambda^{s}, u\right] \tilde{\rho}_{x}, \varpi^{\prime} \tilde{\rho}\right)+\left(\left[\Lambda^{s}, \rho-\bar{\rho}\right] u_{x}, \varpi^{\prime} \tilde{\rho}\right)+\left(\left[\Lambda^{s}, \varpi^{\prime}(\rho)-\varpi(\bar{\rho})\right] \tilde{\rho}_{x}, \rho u\right)+\left(\left[\Lambda^{s}, u\right] u_{x}, \rho u\right)$.

Using (85) and (86) we have

$$
\left|\left(\left[\Lambda^{s}, u\right] \tilde{\rho}_{x}, \varpi^{\prime} \tilde{\rho}\right)\right| \lesssim\left(\left\|\tilde{\rho}_{x}\right\|_{H^{s-1}}+\|u\|_{H^{s}}\right)\|\tilde{\rho}\|_{H^{s}},
$$

where the multiplicative constant depend on $\|W\|_{W^{1, \infty}}$. Doing the same for all the terms we obtain that

As in (106), we obtain

$$
|I| \lesssim\|W\|_{H^{s}}^{2} \lesssim \tilde{E}(W)
$$

$$
|I I| \lesssim \tilde{E}(W)
$$

To estimate $I I I$, we use (87) and (90) to obtain that

$$
\|F\|_{H^{s}} \lesssim\|\psi\|_{H^{s-1}}+\|\tilde{\rho}\|_{H^{s-1}}\left(\|\psi\|_{L^{\infty}}+\|\psi\|_{L^{1}}\right),
$$

where $\psi=\left(\rho^{2} \mathscr{A}^{\prime}\right)^{\prime} u_{x}^{2}+\left(\rho^{\prime} \mathscr{V}^{\prime \prime} / \mathscr{A}^{\prime}\right)^{\prime} \mathscr{A}^{\prime 2} \rho_{x}^{2}$. Using (84), (86) one obtain that $\|\psi\|_{H^{s-1}} \lesssim$ $\|W\|_{H^{s}}$. Due to the conservation of the energy (81), the quantity $\|W\|_{H^{1}}$ is bounded, then $\|\psi\|_{L^{1}}$ is also bounded. Using that $\|W\|_{W^{1, \infty}}$ is bounded, we obtain that $\|\psi\|_{L^{\infty}}$ is also bounded. The inequality (118) then becomes

$$
\|F\|_{H^{s}} \lesssim\|W\|_{H^{s}}
$$

which implies that

$$
|I I I| \lesssim\|W\|_{H^{s}}^{2} \lesssim \tilde{E}(W)
$$

The conservation of the mass (79) implies that $\left\|\rho_{t}\right\|_{L^{\infty}}=\left\|(\rho u)_{x}\right\|_{L^{\infty}}$ which is bounded. Then

$$
|I V| \lesssim\|W\|_{H^{s}}^{2} \lesssim \tilde{E}(W)
$$

The equations (116), (117), (119), (120) and (115) imply that

$$
\tilde{E}(W)_{t} \lesssim \tilde{E}(W)
$$


Gronwall's lemma implies that $\tilde{E}(W)$ does not blow-up in finite time. This ends the proof of the blow-up criteria (82).

\section{A Generalised two-COMPOnEnt Hunter-SAXton SyStem}

We have introduced the Sturm-Louville operator $\mathcal{L}_{\rho}=\rho-2 \epsilon \partial_{x} \rho \mathscr{A}^{\prime} \partial_{x}$ and its inverse $\mathcal{G}_{\rho}=\left[1-2 \epsilon \rho^{-1} \partial_{x} \rho \mathscr{A}^{\prime} \partial_{x}\right]^{-1} \rho^{-1}$. At high frequencies, the operator $\partial_{x} \mathcal{G}_{\rho} \partial_{x}$ obviously behaves like

$$
\partial_{x} \mathcal{G}_{\rho} \partial_{x} \sim-\frac{1}{2} \epsilon^{-1}\left(\rho \mathscr{A}^{\prime}\right)^{-1} .
$$

Thus, differentiating with respect of $x$ the equation (55) and considering the high-frequency approximation (122), the rbE equations become the system of equations

$$
\begin{aligned}
\rho_{t}+[\rho u]_{x} & =0 \\
{\left[u_{t}+u u_{x}+\varpi_{x}\right]_{x} } & =\left(1+\frac{\rho \mathscr{A}^{\prime \prime}}{2 \mathscr{A}^{\prime}}\right) u_{x}^{2}+\left(\frac{\left(\rho \mathscr{V}^{\prime \prime}\right)^{\prime}}{2 \rho}-\frac{\mathscr{V}^{\prime \prime} \mathscr{A}^{\prime \prime}}{2 \mathscr{A}^{\prime}}\right) \rho_{x}^{2},
\end{aligned}
$$

that is a two-component generalisation of the Hunter-Saxton equation [25]. Smooth solutions of (123), (124) satisfy the energy equation

$$
\left[\rho \mathscr{A}^{\prime} u_{x}^{2}+\mathscr{A}^{\prime} \mathscr{V}^{\prime \prime} \rho_{x}^{2}\right]_{t}+\left[\left(\rho \mathscr{A}^{\prime} u_{x}^{2}+\mathscr{A}^{\prime} \mathscr{V}^{\prime \prime} \rho_{x}^{2}\right) u+2 \rho \mathscr{A}^{\prime} \mathscr{V}^{\prime \prime} \rho_{x} u_{x}\right]_{x}=0
$$

There are several generalisations of the Hunter-Saxton equation in the literature, including two-component generalisations. The generalisation (123)-(124) is apparently new and it deserves to be studied since it is a simpler system than rbE, being somehow an asymptotic approximation.

This generalised Hunter-Saxton (gHS) system of equations is to rbE what the HunterSaxton equation [25] is to the dispersionless Camassa-Holm equation [10], i.e., a "high frequency limit". Since the Hunter-Saxton equation is integrable [26], it is of interest to check if this property is shared with the gHS. It should be noted that equation (124) corresponds to $\mathscr{R}=0$, as easily seen considering (52). From a physical viewpoint, the Euler equations describe the "outer" part of a shock, while the gHS equations describe its "inner" structure; the rbE equations being an unification of these two (outer and inner ) systems.

Integrating (124) with respect to $x$, we obtain

$$
\begin{aligned}
\rho_{t}+[\rho u]_{x} & =0, \\
u_{t}+u u_{x}+\varpi_{x} & =\partial_{x}^{-1}\left\{\left(1+\frac{\rho \mathscr{A}^{\prime \prime}}{2 \mathscr{A}^{\prime}}\right) u_{x}^{2}+\left(\frac{\left(\rho \mathscr{V}^{\prime \prime}\right)^{\prime}}{2 \rho}-\frac{\mathscr{V}^{\prime \prime} \mathscr{A}^{\prime \prime}}{2 \mathscr{A}^{\prime}}\right) \rho_{x}^{2}\right\}+g(t),
\end{aligned}
$$

where $\left(\partial_{x}^{-1} f\right)(x) \stackrel{\text { def }}{=} \int_{0}^{x} f(y) \mathrm{d} y$ and $g(t)=u_{t}(t, 0)+u(t, 0) u_{x}(t, 0)+\varpi^{\prime}(\rho(t, 0)) \rho_{x}(t, 0)$.

In the case $\varpi^{\prime} \equiv 0$, the proof of local well-posedness of (126), (127) can be done by using Kato's theorem [28] as in [37, 38, 40, 50]. Following the proof of Theorem 1 and using the inequality

$$
\left\|\partial_{x}^{-1} f\right\|_{H^{s+1}([0,1])} \lesssim\|f\|_{H^{s}([0,1])} \quad \forall s \geqslant 0
$$


one can prove the following theorem

Theorem 3. Let $\tilde{m} \geqslant s \geqslant 2, P, \mathscr{A} \in C^{\tilde{m}+4}(] 0,+\infty[)$ such that $P^{\prime}(\rho)>0, \mathscr{A}^{\prime}(\rho)>0$ for $\rho>0$. Let also $W_{0} \in H^{s}([0,1])$ be a periodic initial data satisfying $\inf _{x \in[0,1]} \rho_{0}(x)>\rho^{*}$ and $g \in C\left(\left[0,+\infty[)\right.\right.$, then there exist $T>0$ and a unique periodic solution $W \in C\left([0, T], H^{s}\right) \cap$ $C^{1}\left([0, T], H^{s-1}\right)$ of $(126)$, (127) satisfying the non-emptiness condition $\inf _{x \in[0,1]} \rho(t, x)>$ 0 and the conservation of the energy

$$
\frac{\mathrm{d}}{\mathrm{d} t} \int_{0}^{1}\left(\rho \mathscr{A}^{\prime} u_{x}^{2}+\mathscr{A}^{\prime} \mathscr{V}^{\prime \prime} \rho_{x}^{2}\right) \mathrm{d} x=0
$$

Moreover, the maximal existence time $T_{\max }<+\infty$, then

$$
\lim _{t \rightarrow T_{\max }}\left\|W_{x}\right\|_{L^{\infty}}=+\infty .
$$

Remark 4. The system (126), (127) do not change if $\mathscr{A}$ is replaced by $-\mathscr{A}$. Then, the result of Theorem 3 holds in the case $\mathscr{A}^{\prime}(\rho)<0$.

\section{REMARKS ON A SPECIAL REgULARISION}

As proved in [36], the solutions given by Theorem 1 do not hold for all time in general. An inspiring way to obtain global (in time) weak solutions, is to use an equivalent semilinear system of ordinary differential equations as in $[8,9,20,48]$. In this case, the lemma 3 is not enough, and an explicit formula of the operator $\mathcal{L}_{\rho}^{-1}$ is needed.

At this stage, the regularising factor $\mathscr{A}$ can be chosen freely, provided that $\mathscr{A}^{\prime}>0$. Here, we investigate further the special choice

$$
\mathscr{A}(\rho)=-A \bar{\rho} / \rho,
$$

where $A>0$ is a constant. We show in this section that with this special choice of $\mathscr{A}$, a formula of $\mathcal{L}_{\rho}^{-1}$ can be obtained, and the rbE system can be simplified.

The Sturm-Liouville operator becomes

$$
\mathcal{L}_{\rho}=\rho-2 \epsilon A \bar{\rho} \partial_{x} \rho^{-1} \partial_{x}=\rho\left[1-2 \epsilon A \bar{\rho} \rho^{-1} \partial_{x} \rho^{-1} \partial_{x}\right],
$$

so its inverse is

$$
\mathcal{G}_{\rho}=\left[1-2 \epsilon A \bar{\rho} \rho^{-1} \partial_{x} \rho^{-1} \partial_{x}\right]^{-1} \rho^{-1}
$$

Similarly, the operator $\mathcal{J}_{\rho}$ becomes

$$
\mathcal{J}_{\rho}=\partial_{x}^{-1} \rho \mathcal{G}_{\rho} \partial_{x}=\left[1-2 \epsilon A \bar{\rho} \rho^{-1} \partial_{x} \rho^{-1} \partial_{x}\right]^{-1} .
$$

This special choice for $\mathscr{A}$ suggests the change of independent variables $(t, x) \mapsto(\tau, \xi)$ with

$$
\tau \stackrel{\text { def }}{=} t, \quad \xi \stackrel{\text { def }}{=} \int \rho(t, x) \mathrm{d} x,
$$

i.e., $\xi$ is a density potential (defined modulo an arbitrary function of $t$ ). After one spacial integration, the equation (42) for the mass conservation yields

$$
\xi_{t}+u \xi_{x}=K(t) \equiv 0,
$$


$K(t)$ being an arbitrary function of $t$ (an integration 'constant') that can be set to zero without loss of generality, thus providing a gauge condition for $\xi$ (i.e., $\xi$ is no longer defined modulo an arbitrary function of $t$ ). Thus, with this change of independent variables, the differentiation operators become

$$
\begin{aligned}
\partial_{x} \mapsto \rho \partial_{\xi}, \quad \partial_{\rho} & \mapsto\left[1-2 \epsilon A \bar{\rho} \partial_{\xi}^{2}\right]^{-1}, \quad \mathcal{G}_{\rho} \partial_{x} \mapsto \partial_{\rho} \partial_{\xi}, \\
\partial_{t} & \mapsto \partial_{\tau}-\rho u \partial_{\xi}, \quad \partial_{t}+u \partial_{x} \mapsto \partial_{\tau},
\end{aligned}
$$

and the regularising term, together with (131), becomes

$$
\mathscr{R}=A \bar{\rho} \mathfrak{J} *\left\{\left(\rho \mathscr{V}^{\prime \prime \prime}+3 \mathscr{V}^{\prime \prime}\right) \rho_{\xi}^{2}\right\}, \quad \mathfrak{J}(\xi) \stackrel{\text { def }}{=} \frac{1}{2 \sqrt{2 \epsilon A \bar{\rho}}} \exp \left(\frac{-|\xi|}{\sqrt{2 \epsilon A \bar{\rho}}}\right),
$$

where an asterix denotes a convolution product, i.e., $\mathfrak{J}(\xi) * f(\xi) \stackrel{\text { def }}{=} \int_{-\infty}^{\infty} \mathfrak{J}(\xi-\tilde{\rho}) f(\tilde{\rho}) \mathrm{d} \tilde{\rho}=$ $\int_{-\infty}^{\infty} \mathfrak{J}(\tilde{\rho}) f(\xi-\tilde{\rho}) \mathrm{d} \tilde{\rho}$ for any function $f$. Note that $\mathfrak{J}$ is the pseudo-differential operator $\mathcal{J}_{\rho}$ rewritten as an integral operator, because it is more convenient when applied to weakly regular functions.

With $(\tau, \xi)$ as independent variables, the mass and momentum equations, respectively (42) and (48), become

$$
\rho_{\tau}+\rho^{2} u_{\xi}=0, \quad u_{\tau}+\left[\rho \mathscr{V}^{\prime}-\mathscr{V}+\epsilon \mathscr{R}\right]_{\xi}=0,
$$

with $\mathscr{R}$ given by (139). Denoting $v \stackrel{\text { def }}{=} 1 / \rho$ the specific volume, the system (140) becomes

$$
v_{\tau}=u_{\xi}, \quad u_{\tau}=\left[\frac{\mathrm{d}(v \mathscr{V})}{\mathrm{d} v}+\epsilon A \bar{\rho} \mathfrak{J} *\left\{\frac{\mathrm{d}^{3}(v \mathscr{V})}{\mathrm{d} v^{3}} v_{\xi}^{2}\right\}\right]_{\xi} .
$$

Eliminating $u$ between these two relations, we obtain

$$
v_{\tau \tau}-\left[\frac{\mathrm{d}(v \mathscr{V})}{\mathrm{d} v}\right]_{\xi \xi}=\epsilon A \bar{\rho} \partial_{\xi}^{2} \mathfrak{J} *\left\{\frac{\mathrm{d}^{3}(v \mathscr{V})}{\mathrm{d} v^{3}} v_{\xi}^{2}\right\}
$$

At high frequencies, this partial differential equation is approximately

$$
v_{\tau \tau}-\left[\frac{\mathrm{d}(v \mathscr{V})}{\mathrm{d} v}\right]_{\xi \xi} \approx-\frac{\mathrm{d}^{3}(v \mathscr{V})}{\mathrm{d} v^{3}} \frac{v_{\xi}^{2}}{2},
$$

that can be rewritten

$$
v_{\tau \tau}-\frac{\mathrm{d}^{2}(v \mathscr{V})}{\mathrm{d} v^{2}} v_{\xi \xi}=\frac{\mathrm{d}^{3}(v \mathscr{V})}{\mathrm{d} v^{3}} \frac{v_{\xi}^{2}}{2},
$$

that is a proper hyperbolic partial differential equation if

$$
\frac{\mathrm{d}^{2}(v \mathscr{V})}{\mathrm{d} v^{2}}>0
$$

Introducing the velocity $c(v) \stackrel{\text { def }}{=} \sqrt{\mathrm{d}^{2}(v \mathscr{V}) / \mathrm{d} v^{2}}$, the equation (144) is rewritten

$$
v_{\tau \tau}-c(v)^{2} v_{\xi \xi}=c(v) c^{\prime}(v) v_{\xi}^{2}
$$

an equation appearing in the theory of liquid crystals, for which smooth solution break down in finite time [19]. 


\section{Conclusion and perspectives}

In this paper, we have introduced the regularised barotropic Euler system (5), inspired by the Hamiltonian regularisation of the shallow water (Saint-Venant) system with a constant depth introduced in [13]. The latter work is generalised in two ways: (i) considering a more general equation (i.e., barotropic Euler); (ii) introducing a family of regularisations (involving an arbitrary function $\mathscr{A}(\rho)$ ).

For this system - and also for the periodic generalised two-component Hunter-Saxton system (6) - we prove the local (in time) well-posedness in $H^{s}$ for $s \geqslant 2$ and a blow-up criteria. As proven by Liu et al. [36], those solutions do not exist for all time, in general.

An interesting question that remains open is: Due to the energy equations (51) and (125), do global weak solutions exist in $H^{1}$ (or in $\dot{H}^{1}$ for (6))? Two possibilities, that have been used for the Camassa-Holm equation, may also work for the systems introduced in the present paper, i.e., using a vanishing viscosity [21] or using a semi-linear equivalent system $[8,9,20,48]$. Another interesting problem is the study of the limiting cases $\varepsilon \rightarrow 0$ and $\varepsilon \rightarrow \infty$ as in $[22,23]$.

\section{Appendix A. Isentropic Flows}

Isentropic motions obey the equation of state

$$
\rho / \bar{\rho}=(P / \bar{P})^{1 / \gamma}, \quad P / \bar{P}=(\rho / \bar{\rho})^{\gamma},
$$

where $\bar{\rho}$ and $\bar{P}$ are positive constants characterising the fluid physical properties at the rest state, and $\gamma \stackrel{\text { def }}{=} C_{\mathrm{p}} / C_{\mathrm{v}}$ is the (constant) ratio of specific heats $C_{\mathrm{p}}$ and $C_{\mathrm{v}}$. It should be noted that the constitutive relation (147) gauges the pressure field, so zero pressure level can no longer be chosen arbitrarily without loss of generality. For these isentropic motions, we have if $\gamma \neq 1$ (taking $\varpi(\bar{P})=0$ )

$$
\begin{aligned}
\varpi & =\int\left(\frac{\bar{P}}{P}\right)^{\frac{1}{\gamma}} \frac{\mathrm{d} P}{\bar{\rho}}=\bar{\varpi} \frac{(P / \bar{P})^{\frac{\gamma-1}{\gamma}}-1}{\gamma-1}=\bar{\varpi} \frac{(\rho / \bar{\rho})^{\gamma-1}-1}{\gamma-1}, \\
\frac{\mathscr{V}}{\bar{P}} & =\frac{\gamma}{\gamma-1}\left[\frac{1}{\gamma}\left(\frac{\rho}{\bar{\rho}}\right)^{\gamma}-\frac{\rho}{\bar{\rho}}\right]=\frac{\gamma}{\gamma-1}\left[\frac{1}{\gamma} \frac{P}{\bar{P}}-\frac{\rho}{\bar{\rho}}\right],
\end{aligned}
$$

where $\bar{\varpi} \stackrel{\text { def }}{=} \gamma \bar{P} / \bar{\rho}$, thence

$$
P / \bar{P}=\left(c_{s}^{2} / \bar{\varpi}\right)^{\gamma /(\gamma-1)}, \quad c_{s}^{2} / \bar{\varpi}=1+(\gamma-1)(\varpi / \bar{\varpi}) .
$$

In the limiting case $\gamma \rightarrow 1$ (isothermal motions), these relations become

$$
c_{s}^{2}=\bar{\varpi}=\frac{\bar{P}}{\bar{\rho}}, \quad \frac{\rho}{\bar{\rho}}=\frac{P}{\bar{P}}=\exp \left(\frac{\varpi}{\bar{\varpi}}\right), \quad \frac{\mathscr{V}}{\bar{P}}=\frac{\rho}{\bar{\rho}} \log \left|\frac{\rho}{\bar{\rho}}\right|-\frac{\rho}{\bar{\rho}},
$$

so the speed of sound is constant while the density is not. The special case $\gamma=1$ is relevant for applications in oceanography because for seawater (at salinity $35 \mathrm{~g} / \mathrm{kg}$ and atmospheric pressure) $\gamma \approx 1.0004$ at $0^{\circ} \mathrm{C}$ and $\gamma \approx 1.0106$ at $20^{\circ} \mathrm{C}$ [30]. 


\section{REFERENCES}

[1] S. Alinhac. Blowup for nonlinear hyperbolic equations. Springer Science $\&$ Business Media, Vol. 17, 2013.

[2] S. Alinhac and P. Gérard. Pseudo-differential operators and the Nash-Moser theorem. American Mathematical Soc., Vol. 82, 2007.

[3] H. S. Bhat and R. C. Fetecau. A Hamiltonian regularization of the Burgers equation. J. Nonlinear Sci., 16(6):615-638, 2006.

[4] H. S. Bhat and R. C. Fetecau. The Riemann problem for the Leray-Burgers equation. J. Diff. Eq., 246:3957-3979, 2009.

[5] H. S. Bhat and R. C. Fetecau. On a regularization of the compressible Euler equations for an isothermal gas. J. Math. Anal. Appl., 358(1):168-181, 2009.

[6] H. S. Bhat, R. C. Fetecau, and J. Goodman. A Leray-type regularization for the isentropic Euler equations. Nonlinearity, 20(9):2035-2046, 2007.

[7] S. Bianchini and A. Bressan. Vanishing viscosity solutions of nonlinear hyperbolic systems. Annals of mathematics, pages 223-342, 2005.

[8] A. Bressan and A. Constantin. Global conservative solutions of the Camassa-Holm equation. Arch. Rat. Mech. Anal., 183(2):215-239, 2007.

[9] A. Bressan and A. Constantin. Global dissipative solutions of the Camassa-Holm equation. Anal. \& Appl., 5(1):1-27, 2007.

[10] R. Camassa and D. D. Holm. An integrable shallow water equation with peaked solitons. Phys. Rev. Lett., 71(11):1661-1664, 1993.

[11] R. Camassa, P.-H. Chiu, L. Lee, and T. W. H. Sheu. Viscous and inviscid regularizations in a class of evolutionary partial differential equations. J. Comp. Phys., 229: 6676-6687, 2010.

[12] D. Clamond and D. Dutykh. Fast accurate computation of the fully nonlinear solitary surface gravity waves. Comp. Ef Fluids, 84:35-38, 2013.

[13] D. Clamond and D. Dutykh. Non-dispersive conservative regularisation of nonlinear shallow water (and isentropic Euler) equations. Comm. Nonlin. Sci. Numer. Simul., 55:237-247, 2018.

[14] D. Clamond, D. Dutykh, and D. Mitsotakis. Hamiltonian regularisation of shallow water equations with uneven bottom. Journal of Physics A: Mathematical and Theoretical, 52(42):42LT01, 2019.

[15] A. Constantin and L. Molinet. The initial value problem for a generalized Boussinesq equation. Differential and Integral equations., 15(9):1061-1072, 2002.

[16] M. G. Crandall and P.-L. Lions. Viscosity solutions of Hamilton-Jacobi equations. Transactions of the American mathematical society, 277(1):1-42, 1983.

[17] C.M. Dafermos. Hyperbolic Conservation Laws in Continuum Physics. Springer, Vol. 325, 2016.

[18] F. V. Dolzhansky. Fundamentals of geophysical hydrodynamics. Springer, 2003.

[19] R. T. Glassey, J. K. Hunter and Y. Zheng. Singularities and oscillations in a nonlinear variational wave equation. Singularities and Oscillations, IMA 91, 37-60. The IMA Volumes in Mathematics and its Applications book series, Springer. 
[20] K. Grunert, H. Holden, and X. Raynaud. Global solutions for the two-component Camassa-Holm system. Communications in Partial Differential Equations. 37(12): 2245-2271, 2012.

[21] C. Guan, and Z. Yin. Global weak solutions for a modified two-component CamassaHolm equation. Annales de l'Institut Henri Poincare C, Non Linear Analysis. 28(4): 623-641, 2011.

[22] B. Guelmame. On a Hamiltonian regularization of scalar conservation laws. hal02512810, 2020.

[23] B. Guelmame, S. Junca, D. Clamond, and R. Pego. Global weak solutions of a Hamiltonian regularised Burgers equation. hal-02478872, 2020.

[24] B. T. Hayes and P. G. LeFloch. Nonclassical shocks and kinetic relations: strictly hyperbolic systems. SIAM J. Math. Anal., 31(5):941-991, 2000.

[25] J. K. Hunter and R. Saxton. Dynamics of director fields. SIAM J. Appl. Math., 51 (6):1498-1521, 1991.

[26] J. K. Hunter and Y. Zheng. On a completely integrable nonlinear hyperbolic variational equation. Physica D, 79(2-4):361-386, 1994.

[27] S. Israwi. Large time existence for 1D Green-Naghdi equations. Nonlinear Analysis, 74(1):81-93, 2011.

[28] T. Kato. Quasi-linear equations of evolution, with applications to partial differential equations. Spectral theory and differential equations., Springer :25-70, 1975.

[29] T. Kato and G. Ponce. Commutator estimates and the Euler and Navier-Stokes equations. Communications on Pure and Applied Mathematics., 41(7):891-907, 1988.

[30] G. W. C. Kaye and T. H. Laby. Tables of Physical and Chemical Constants. Longman Sci. \& Tech, 16th edition, 1995.

[31] C. I. Kondo and P. G. LeFloch. Zero diffusion-dispersion limits for scalar conservation laws. SIAM journal on mathematical analysis, 33(6):1320-1329, 2002.

[32] P. D. Lax. Shock waves and entropy. In Contributions to nonlinear functional analysis, 603-634, 1971.

[33] P. D. Lax and C. D. Levermore. The small dispersion limit of the KdV equations I. Comm. Pure Appl. Math., 3:253-290, 1983.

[34] P. D. Lax and C. D. Levermore. The small dispersion limit of the KdV equations II. Comm. Pure Appl. Math., 5:571-593, 1983.

[35] P. D. Lax and C. D. Levermore. The small dispersion limit of the KdV equations III. Comm. Pure Appl. Math., 6:809-829, 1983.

[36] J.-L. Liu, R. L. Pego, and Y. Pu. Well-posedness and derivative blow-up for a dispersionless regularized shallow water system. Nonlinearity., 32(11):4346-4376, 2019.

[37] J. Liu, and Z. Yin. On the Cauchy problem of a periodic 2-component $\mu$-HunterSaxton system. Nonlinear Analysis: Theory, Methods \&f Applications., 75(1): 131$142,2012$.

[38] J. Liu, and Z. Yin. On the Cauchy problem of a weakly dissipative $\mu$-Hunter-Saxton equation Annales de l'Institut Henri Poincare (C) Non Linear Analysis., Vol. 31. 267-279 2014. 
[39] A. Majda. Compressible fluid flow and systems of conservation laws in several space variables, volume 53. Springer Science \& Business Media, 2012.

[40] B. Moon and Y. Liu. Wave breaking and global existence for the generalized periodic two-component Hunter-Saxton system. J. Diff. Eq., 253(1):319-355, 2012.

[41] G. J. Norgard and K. Mohseni. An examination of the homentropic Euler equations with averaged characteristics. J. Diff. Eq., 248:574-593, 2010.

[42] G. J. Norgard and K. Mohseni. A new potential regularization of the one-dimensional Euler and homentropic Euler equations. Multiscale Model. Simul., 8(4):1212-1243, 2010.

[43] Y. Nutku. On a new class of completely integrable nonlinear wave equations. II. Multi-Hamiltonian structure. J. Math. Phys., 28(11):2579-2585, 1987.

[44] Y. Pu. Weakly singular waves and blow-up for a regularization of the shallow-water system. PhD thesis, Carnegie Mellon University, 2018.

[45] Y. Pu, R. L. Pego, D. Dutykh, and D. Clamond. Weakly singular shock profiles for a non-dispersive regularization of shallow-water equations. Comm. Math. Sci., 16(5): 1361-1378, 2018.

[46] J. Serrin. Mathematical principles of classical fluid mechanics. In S. Flugge and C. Truedell, editors, Fluid Dynamics I, volume VIII/1 of Encyclopaedia of Physics, pages 125-263. Springer-Verlag, 1959.

[47] J. VonNeumann and R. D. Richtmyer. A method for the numerical calculation of hydrodynamic shocks. J. Appl. Phys., 21(3):232-237, 1950.

[48] Y. Wang, J. Huang, and L. Chen. Global conservative solutions of the two-component Camassa-Holm shallow water system. International Journal of Nonlinear Science., 9 (3):379-384, 2010.

[49] W. Wagner and A. Pruß. The IAPWS formulation 1995 for the thermodynamic properties of ordinary water substance for general and scientific use. J. Phys. Chem. Ref. Data, 31(2):387-535, 2002.

[50] Z. Yin. On the Structure of Solutions to the Periodic Hunter-Saxton Equation. SIAM journal on mathematical analysis, 36(1):272-283, 2004.

(Billel Guelmame) LJAD, Inria \& CNRS, Université Côte D'Azur, France.

Email address: billel.guelmame@univ-cotedazur.fr

(Didier Clamond) LJAD, CNRS, Université Côte D'Azur, France.

Email address: didier.clamond@univ-cotedazur.fr

(Stéphane Junca) LJAD, Inria \& CNRS, Université Côte D’Azur, France.

Email address: stephane.junca@univ-cotedazur.fr 